Mathematical Modelling and Analysis

Volume 21 Number 6, November 2016, 719-740

http://dx.doi.org/10.3846/13926292.2016.1214930

(C) Vilnius Gediminas Technical University, 2016
Publisher: Taylor\&Francis and VGTU

http://www.tandfonline.com/TMMA

ISSN: $1392-6292$

eISSN: $1648-3510$

\title{
Deviation of the Error Estimation for Second Order Fredholm-Volterra Integro Differential Equations
}

\section{Reza Parvaz ${ }^{a}$, Mohammad Zarebnia ${ }^{a}$ and Amir Saboor Bagherzadeh ${ }^{b}$}

${ }^{a}$ Department of Mathematics, University of Mohaghegh Ardabili, 56199-11367 Ardabil, Iran.

${ }^{b}$ Institute of Structural Mechanics, Bauhaus Universität-Weimar, Marienstrasse 15, 99423 Weimar, Germany.

E-mail(corresp.): zarebnia@uma.ac.ir

E-mail: rparvaz@uma.ac.ir

E-mail: saboorbagherzadeh.a@gmail.com

Received January 31, 2016; revised July 14, 2016; published online November 15, 2016

\begin{abstract}
In this paper we study the deviation of the error estimation for the second order Fredholm-Volterra integro-differential equations. We prove that for $m$ degree piecewise polynomial collocation method, our method provides $\mathcal{O}\left(h^{m+1}\right)$ as the order of the deviation of the error. Also numerical results in the final section are included to confirm the theoretical results.
\end{abstract}

Keywords: deviation of the error, finite difference, exact finite difference, integrodifferential.

AMS Subject Classification: 41A25; 45J05; 65N35.

\section{Introduction}

In this paper we consider the second order Fredholm-Volterra integrodifferential (SFVID) equations as follows

$$
\begin{aligned}
& y^{\prime \prime}(t)=F\left(t, y(t), y^{\prime}(t), z_{\mathbf{f}}[y](t), z_{\mathbf{v}}[y](t)\right), \quad t \in I:=[a, b], \\
& y(a)=r_{1}, \quad y(b)=r_{2},
\end{aligned}
$$

where

$$
\begin{aligned}
& z_{\mathbf{f}}[y](t)=\int_{a}^{b} K_{\mathbf{f}}\left(t, s, y(s), y^{\prime}(s), y^{\prime \prime}(s)\right) d s \\
& z_{\mathbf{v}}[y](t)=\int_{a}^{t} K_{\mathbf{v}}\left(t, s, y(s), y^{\prime}(s), y^{\prime \prime}(s)\right) d s
\end{aligned}
$$


and $a, b, r_{1}, r_{2} \in R=(-\infty, \infty)$. $W$ and $S$ are defined as follows

$$
\begin{aligned}
& W:=\left\{\left(t, y, y^{\prime}, z_{\mathbf{f}}, z_{\mathbf{v}}\right) ; t \in I \quad \text { and } y, y^{\prime}, z_{\mathbf{f}}, z_{\mathbf{v}} \in \mathbb{R}\right\} \\
& S:=\left\{\left(t, s, u, u^{\prime}, u^{\prime \prime}\right) ; t, s \in I \quad \text { and } u, u^{\prime}, u^{\prime \prime} \in \mathbb{R}\right\} .
\end{aligned}
$$

In this paper we shall assume that $F$ is uniformly continuous in $W$. Also we assume that $z_{\mathbf{f}}[y](t), z_{\mathbf{v}}[y](t)$ are uniformly continuous in $S$. We say that $z_{\mathbf{f}}[y](t)$ and $z_{\mathbf{v}}[y](t)$ are linear if we can write $z_{\mathbf{f}}[y](t)$ and $z_{\mathbf{v}}[y](t)$ as

$$
z_{\mathbf{f}}[y](t)=\sum_{l=0}^{2} \int_{a}^{b} \Lambda_{l, \mathbf{f}}(t, s) y^{(l)}(s) d s, \quad z_{\mathbf{v}}[y](t)=\sum_{l=0}^{2} \int_{a}^{t} \Lambda_{l, \mathbf{v}}(t, s) y^{(l)}(s) d s,
$$

where $\Lambda_{l, j}(t, s) \quad(l=0,1,2 \& j=\mathbf{f}, \mathbf{v})$ are sufficiently smooth in $J:=\{(t, s)$; $t, s \in I\}$. Also we say that $F$ is linear if we can write it as

$$
F\left(t, y(t), y^{\prime}(t), z_{\mathbf{f}}[y](t), z_{\mathbf{v}}[y](t)\right)=\sum_{l=1}^{2} a_{l}(t) y^{(2-l)}(t)+\sum_{l=\mathbf{f}, \mathbf{v}} z_{l}[y](t)+a_{3}(t) .
$$

In the nonlinear case we assume that $F\left(t, y, y^{\prime}, z_{\mathbf{f}}, z_{\mathbf{v}}\right), F_{l}\left(t, y, y^{\prime}, z_{\mathbf{f}}, z_{\mathbf{v}}\right)$ for any $l=t, y, y^{\prime}, z_{\mathbf{f}}, z_{\mathbf{v}}$ are Lipschitz-continuous. When $z_{\mathbf{f}}[y](t)$ and $z_{\mathbf{v}}[y](t)$ are nonlinear we assume that $K_{j}\left(t, s, u, u^{\prime}, u^{\prime \prime}\right)$ and $\left(K_{j}\right)_{l}\left(t, s, u, u^{\prime}, u^{\prime \prime}\right) \quad(j=$ $\left.\mathbf{f}, \mathbf{v} \& l=u, u^{\prime}, u^{\prime \prime}\right)$ are Lipschitz-continuous. We say SFVID equation with boundary condition (1.2) is linear if we can write (1.1) as follows

$$
y^{\prime \prime}(t)=a_{1}(t) y^{\prime}(t)+a_{2}(t) y(t)+a_{3}(t)+z_{\mathbf{f}}[y](t)+z_{\mathbf{v}}[y](t), \quad t \in[a, b]
$$

with linear $z_{\mathbf{f}}[y](t)$ and $z_{\mathbf{v}}[y](t)$. Also, in the linear case we assume that $a_{i}(t)$, $i=1,2,3$ are sufficiently smooth in $I$. In this paper we use the defect correction principle, more details about this can be found in $[4,9]$. The piecewise polynomial collocation method for integro-differential equations can be found in $[5,6,7,8]$. Also other methods for the integro-differential equations are studied in $[11,12]$. The deviation of the error estimation for linear and nonlinear first and second order boundary value problem is studied in $[1,2,3]$. The error estimation based on locally weighted defect that we will use in this manuscript, has been introduced in $[1,3]$.

The rest of this paper is organized as follows. In Section 2, the method is described and we introduce some details about the deviation of the error for SFVID. In Section 3, the analysis of the deviation of the error is given. Also the main results of the paper are formulate in Theorems 4-5. In Section 4, we study the special case of SFVID equation. And we show that in this case for $m$ degree piecewise polynomial collocation method, our method provides $\mathcal{O}\left(h^{m+2}\right)$ as the order of the deviation of the error. In Section 5 , we present the numerical experiments that demonstrate our theoretical results. A summary is given at the end of the paper in Conclusion section.

\section{Description of the method}

In this section, we introduce some details about the deviation of the error estimation, collocation method, finite differences and exact difference schemes. 


\subsection{Collocation method}

In the first step we consider $\tau_{i}, \rho_{i}$ as follows

$$
a=\tau_{0}<\tau_{1}<\ldots<\tau_{n}=b,(n \geq 1), \quad 0=\rho_{0}<\rho_{1}<\ldots<\rho_{m}<\rho_{m+1}=1 .
$$

DeFinition 1. In this paper we define

$$
\begin{aligned}
& X_{i}:=\left\{t_{i, j}:=\tau_{i}+\rho_{j} h_{i} ; j=1, \ldots, m\right\}, \quad Z_{n}:=\left\{t_{i, 0}:=\tau_{i} ; i=0, \ldots, n\right\}, \\
& S_{m+1}^{(1)}\left(Z_{n}\right):=\left\{p \in \mathcal{C}^{1}(I) ; p \uparrow\left[\tau_{i}, \tau_{i+1}\right] \in \Pi_{m+1}\left(\left[\tau_{i}, \tau_{i+1}\right]\right)(i=0, \ldots, n-1)\right\},
\end{aligned}
$$

where $h_{i}:=\tau_{i+1}-\tau_{i}$ and $\Pi_{m+1}\left(\left[\tau_{i}, \tau_{i+1}\right]\right)$ is space of real polynomial functions on $\left[\tau_{i}, \tau_{i+1}\right]$ of degree $\leqslant m+1$. Also we define $h$ (the diameter of gird $Z_{n}$ ) and $h^{\prime}$ as

$$
h:=\max \left\{h_{i} ; i=0, \ldots, n-1\right\}, \quad h^{\prime}:=\min \left\{h_{i} ; i=0, \ldots, n-1\right\} .
$$

The set $X(n):=\bigcup_{i=0}^{n-1} X_{i}$ is called the set of collocation points.

In the piecewise polynomial collocation method we are looking to find a $p \in S_{m+1}^{(1)}\left(Z_{n}\right)$ so that (1.1)-(1.2) holds for all $t_{i, j} \in X(n)$. In the collocation method, since always we can not determine exact value for $z_{l}[p](t) \quad(l=\mathbf{f}, \mathbf{v})$, therefore we use the following quadrature method to determine $z_{l}[p]\left(t_{i, j}\right) \quad(l=$ $\mathbf{f}, \mathbf{v})$.

$$
\begin{aligned}
& z_{\mathbf{f}}[p]\left(t_{i, j}\right) \approx \sum_{k=0}^{n-1} \sum_{z=0}^{m+1} \alpha_{k, z} K_{\mathbf{f}}\left(t_{i, j}, t_{k, z}, p\left(t_{k, z}\right), p^{\prime}\left(t_{k, z}\right), p^{\prime \prime}\left(t_{k, z}\right)\right)=: \widetilde{z}_{\mathbf{f}}[p]\left(t_{i, j}\right), \\
& z_{\mathbf{v}}[p]\left(t_{i, j}\right) \approx \sum_{k=0}^{i-1} \sum_{z=0}^{m+1} \alpha_{k, z} K_{\mathbf{v}}\left(t_{i, j}, t_{k, z}, p\left(t_{k, z}\right), p^{\prime}\left(t_{k, z}\right), p^{\prime \prime}\left(t_{k, z}\right)\right)+\left(t_{i, j}-\tau_{i}\right) \\
& \quad \times \sum_{z=0}^{m+1} \beta_{z} K_{\mathbf{v}}\left(t_{i, j}, \bar{t}_{i, j, z}, p\left(\bar{t}_{i, j, z}\right), p^{\prime}\left(\bar{t}_{i, j, z}\right), p^{\prime \prime}\left(\bar{t}_{i, j, z}\right)\right)=: \widetilde{z}_{\mathbf{v}}[p]\left(t_{i, j}\right),
\end{aligned}
$$

where $\bar{t}_{i, j, z}:=\tau_{i}+\rho_{z}\left(t_{i, j}-\tau_{i}\right)$ and

$$
\alpha_{k, z}:=\int_{\tau_{k}}^{\tau_{k+1}} L_{z}^{\left[\tau_{k}, \tau_{k+1}\right]}(s) d s, \quad \beta_{z}:=\int_{0}^{1} L_{z}(s) d s
$$

with

$$
L_{j}(\rho):=\prod_{\substack{i=0 \\ i \neq j}}^{m+1} \frac{\rho-\rho_{i}}{\rho_{j}-\rho_{i}}, \quad L_{j}^{\left[a^{\prime}, b^{\prime}\right]}(\rho):=L_{j}\left(\frac{\rho-a^{\prime}}{b^{\prime}-a^{\prime}}\right), \quad a \leq a^{\prime}<b^{\prime} \leq b .
$$

According to [10] we have the following theorem. 
Theorem 1. (Interpolation Error Theorem) If the function $f$ has an $(n+1)$ st derivative and $P_{n}(x)$ be a polynomial of degree at most $n$ that interpolates $f$ at $n+1$ distinct points $x_{i}(i=0, \ldots, n)$, then for every argument $\bar{x}$ there exists a number $\zeta$ in the smallest interval $I\left[x_{0}, \ldots, x_{n}, \bar{x}\right]$ which contains $\bar{x}$ and all support abscissas $x_{i}$, satisfying

$$
f(\bar{x})-P_{n}(\bar{x})=\frac{w(\bar{x}) f^{(n+1)}(\zeta)}{(n+1) !},
$$

where $w(x):=\left(x-x_{0}\right) \ldots\left(x-x_{n}\right)$.

For the above method we have the following lemma.

Lemma 1. For sufficiently smooth $f$, the following estimate holds

$$
\left|z_{l}[f]\left(t_{i, j}\right)-\widetilde{z}_{l}[f]\left(t_{i, j}\right)\right|=\mathcal{O}\left(h^{m+2}\right), \quad l=\mathbf{f}, \mathbf{v},
$$

where $\widetilde{z}_{\mathbf{f}}[\cdot]\left(t_{i, j}\right)$ and $\widetilde{z}_{\mathbf{v}}[\cdot]\left(t_{i, j}\right)$ are defined in (2.1)-(2.2).

Proof. For nonlinear $\widetilde{z}_{\mathbf{f}}[\cdot]$, we can write

$$
\begin{gathered}
z_{\mathbf{f}}[f]\left(t_{i, j}\right)-\widetilde{z}_{\mathbf{f}}[f]\left(t_{i, j}\right)=\int_{a}^{b} K_{\mathbf{f}}\left(t_{i, j}, s, f(s), f^{\prime}(s), f^{\prime \prime}(s)\right) d s-\sum_{k=0}^{n-1} \sum_{z=0}^{m+1} \alpha_{k, z} \\
\times K_{\mathbf{f}}\left(t_{i, j}, t_{k, z}, f\left(t_{k, z}\right), f^{\prime}\left(t_{k, z}\right), f^{\prime \prime}\left(t_{k, z}\right)\right)=\sum_{k=0}^{n-1} \int_{\tau_{k}}^{\tau_{k+1}} I_{1}(s) d s .
\end{gathered}
$$

By using Interpolation error theorem, we can get that $I_{1}=\mathcal{O}\left(h^{m+2}\right)$. Then we can rewrite (2.4) as follows

$$
z_{\mathbf{f}}[f]\left(t_{i, j}\right)-\widetilde{z}_{\mathbf{f}}[f]\left(t_{i, j}\right) \leq n h \mathcal{O}\left(h^{m+2}\right) \leq \frac{h}{h^{\prime}}(b-a) \mathcal{O}\left(h^{m+2}\right)=\mathcal{O}\left(h^{m+2}\right)
$$

Also for $l=\mathbf{v}$, we get

$$
z_{\mathbf{v}}[f]\left(t_{i, j}\right)-\widetilde{z}_{\mathbf{v}}[f]\left(t_{i, j}\right)=\sum_{k=0}^{i-1} \int_{\tau_{k}}^{\tau_{k+1}} I_{2}(s) d s+\left(t_{i, j}-\tau_{i}\right) \int_{0}^{1} I_{3}(s) d s,
$$

where

$$
\begin{aligned}
I_{2}(s):= & K_{\mathbf{v}}\left(t_{i, j}, s, f(s), f^{\prime}(s), f^{\prime \prime}(s)\right) \\
& -\sum_{z=0}^{m+1} L_{z}^{\left[\tau_{k}, \tau_{k+1}\right]}(s) K_{\mathbf{v}}\left(t_{i, j}, t_{k, z}, f\left(t_{k, z}\right), f^{\prime}\left(t_{k, z}\right), f^{\prime \prime}\left(t_{k, z}\right)\right), \\
I_{3}(s):= & K_{\mathbf{v}}\left(t_{i, j}, \breve{t}_{i, j, s}, f\left(\breve{t}_{i, j, s}\right), f^{\prime}\left(\breve{t}_{i, j, s}\right), f^{\prime \prime}\left(\breve{t}_{i, j, s}\right)\right) \\
& -\sum_{z=0}^{m+1} L_{z}(s) K_{\mathbf{v}}\left(t_{i, j}, \bar{t}_{i, j, z}, f\left(\bar{t}_{i, j, z}\right), f^{\prime}\left(\bar{t}_{i, j, z}\right), f^{\prime \prime}\left(\bar{t}_{i, j, z}\right)\right)
\end{aligned}
$$


where $\breve{t}_{i, j, s}:=\tau_{i}+s\left(t_{i, j}-\tau_{i}\right)$. By using Interpolation error theorem, we can say $I_{2}=\mathcal{O}\left(h^{m+2}\right)$ and $I_{3}=\mathcal{O}\left(h^{m+2}\right)$. Then we get

$$
z_{\mathbf{v}}[f]\left(t_{i, j}\right)-\widetilde{z}_{\mathbf{v}}[f]\left(t_{i, j}\right) \leq \frac{h}{h^{\prime}}\left(\tau_{i}-\tau_{0}\right) \mathcal{O}\left(h^{m+2}\right)+\mathcal{O}\left(h^{m+2}\right)=\mathcal{O}\left(h^{m+2}\right),
$$

which completes the proof. Similarly, we can find (2.3) for linear case.

For above collocation method we have the following theorem [5].

Theorem 2. Assume that the SFVID problem (1.1)-(1.2) has a unique and sufficiently smooth solution $y(t)$. Also assume that $p(t)$ is a piecewise polynomial collocation solution of degree $\leq m+1$. Then for sufficiently small $h$, the collocation solution $p(t)$ is well-defined and the following uniform estimates at least hold:

$$
\begin{aligned}
& \left\|y^{(j)}(t)-p^{(j)}(t)\right\|_{\infty}=\mathcal{O}\left(h^{m}\right), j=0,1,2, \\
& \left\|y^{(j)}(t)-p^{(j)}(t)\right\|_{\infty}=\mathcal{O}\left(h^{m+2-j}\right), j=3, \ldots, m+1 .
\end{aligned}
$$

Also in the piecewise polynomial collocation method when $m$ is odd and the nodes $\rho_{i}$ are symmetrically distributed we have

$$
\left\|y^{(j)}(t)-p^{(j)}(t)\right\|_{\infty}=\mathcal{O}\left(h^{m+1}\right), j=0,1
$$

Lemma 2. For linear and nonlinear $z_{l}[\cdot](t)(l=\mathbf{f}, \mathbf{v})$ we have

$$
\left|\widetilde{z}_{l}[p]\left(t_{i, j}\right)-\widetilde{z}_{l}[y]\left(t_{i, j}\right)\right|=\mathcal{O}\left(h^{m}\right), \quad l=\mathbf{f}, \mathbf{v} .
$$

Proof. For linear case by using Lemma 1, Theorem 2 and the Integral mean value theorem, we get

$$
\begin{aligned}
\widetilde{z}_{\mathbf{f}}[p]\left(t_{i, j}\right) & -\widetilde{z}_{\mathbf{f}}[y]\left(t_{i, j}\right)=z_{\mathbf{f}}[e]\left(t_{i, j}\right)+\mathcal{O}\left(h^{m+2}\right) \\
& =\sum_{l=0}^{2} \int_{a}^{b} \Lambda_{l, \mathbf{f}}\left(t_{i, j}, s\right) e^{(l)}(s) d s+\mathcal{O}\left(h^{m+2}\right) \\
& =\sum_{l=0}^{2}(b-a) \Lambda_{l, \mathbf{f}}\left(t_{i, j}, \zeta_{i, j}^{l}\right) \underbrace{e^{(l)}\left(\zeta_{i, j}^{l}\right)}_{\mathcal{O}\left(h^{m}\right)}+\mathcal{O}\left(h^{m+2}\right)=\mathcal{O}\left(h^{m}\right),
\end{aligned}
$$

where $\zeta_{i, j}^{l} \in[a, b]$. Also we can obtain

$$
\begin{aligned}
\widetilde{z}_{\mathbf{v}}[p]\left(t_{i, j}\right) & -\widetilde{z}_{\mathbf{v}}[y]\left(t_{i, j}\right)=z_{\mathbf{v}}[e]\left(t_{i, j}\right)+\mathcal{O}\left(h^{m+2}\right) \\
& =\sum_{l=0}^{2} \int_{a}^{t_{i, j}} \Lambda_{l, \mathbf{v}}\left(t_{i, j}, s\right) e^{(l)}(s) d s+\mathcal{O}\left(h^{m+2}\right) \\
& =\sum_{l=0}^{2}\left(t_{i, j}-a\right) \Lambda_{l, \mathbf{v}}\left(t_{i, j}, \bar{\zeta}_{i, j}^{l}\right) \underbrace{e^{(l)}\left(\bar{\zeta}_{i, j}^{l}\right)}_{\mathcal{O}\left(h^{m}\right)}+\mathcal{O}\left(h^{m+2}\right)=\mathcal{O}\left(h^{m}\right),
\end{aligned}
$$


where $\bar{\zeta}_{i, j}^{l} \in[a, b]$. Also for nonlinear case by using Lemma 1 and the Lipschitz condition for $K_{l}(l=\mathbf{f}, \mathbf{v})$ we can find

$$
\begin{aligned}
& \left|\widetilde{z}_{\mathbf{f}}[y]\left(t_{i, j}\right)-\widetilde{z}_{\mathbf{f}}[p]\left(t_{i, j}\right)\right| \\
& \leq\left|\widetilde{z}_{\mathbf{f}}[y]\left(t_{i, j}\right)-z_{\mathbf{f}}[y]\left(t_{i, j}\right)-\widetilde{z}_{\mathbf{f}}[p]\left(t_{i, j}\right)+z_{\mathbf{f}}[p]\left(t_{i, j}\right)+z_{\mathbf{f}}[y]\left(t_{i, j}\right)-z_{\mathbf{f}}[p]\left(t_{i, j}\right)\right| \\
& \leq\left|\int_{a}^{b}\left(K_{\mathbf{f}}\left(t_{i, j}, s, y(s), y^{\prime}(s), y^{\prime \prime}(s)\right)-K_{\mathbf{f}}\left(t_{i, j}, s, p(s), p^{\prime}(s), p^{\prime \prime}(s)\right)\right) d s\right| \\
& \quad+\mathcal{O}\left(h^{m+2}\right) \leq C \sum_{l=0}^{2} \int_{a}^{b}\left|y^{(l)}(s)-p^{(l)}(s)\right| d s+\mathcal{O}\left(h^{m+2}\right)=\mathcal{O}\left(h^{m}\right), \\
& \quad\left|\widetilde{z}_{\mathbf{v}}[y]\left(t_{i, j}\right)-\widetilde{z}_{\mathbf{v}}[p]\left(t_{i, j}\right)\right| \\
& \leq\left|\widetilde{z}_{\mathbf{v}}[y]\left(t_{i, j}\right)-z_{\mathbf{v}}[y]\left(t_{i, j}\right)-\widetilde{z}_{\mathbf{v}}[p]\left(t_{i, j}\right)+z_{\mathbf{v}}[p]\left(t_{i, j}\right)+z_{\mathbf{v}}[y]\left(t_{i, j}\right)-z_{\mathbf{v}}[p]\left(t_{i, j}\right)\right| \\
& \leq\left|\int_{a}^{t_{i, j}}\left(K_{\mathbf{v}}\left(t_{i, j}, s, y(s), y^{\prime}(s), y^{\prime \prime}(s)\right)-K_{\mathbf{v}}\left(t_{i, j}, s, p(s), p^{\prime}(s), p^{\prime \prime}(s)\right)\right) d s\right| \\
& \quad+\mathcal{O}\left(h^{m+2}\right) \leq C \sum_{l=0}^{2} \int_{a}^{t_{i, j}}\left|y^{(l)}(s)-p^{(l)}(s)\right| d s+\mathcal{O}\left(h^{m+2}\right)=\mathcal{O}\left(h^{m}\right),
\end{aligned}
$$

which completes the proof.

\subsection{Finite difference scheme}

Definition 2. In this subsection we define

$$
\begin{aligned}
& \Delta_{i, j}:=\{(l, k) ; l=0, \ldots, i-1 \& k=0, \ldots, m\} \cup\{(i, k) ; k=0, \ldots, j-1\}, \\
& \mathcal{A}:=\left\{(i, j) ; t_{i, j} \in X(n) \cup Z_{n}\right\}, \mathcal{B}:=\mathcal{A}-\{(0,0),(n, 0)\}, \mathcal{T}:=\mathcal{A}-\{(n, 0)\}
\end{aligned}
$$

Also we define

$$
\delta_{i, j}:=t_{i, j+1}-t_{i, j}, \quad \widehat{\delta}_{i, j}:=\frac{\delta_{i, j-1}+\delta_{i, j}}{2}, \widehat{\alpha}_{i, j}:=\frac{\delta_{i, j-1}}{\widehat{\delta}_{i, j}}, \widehat{\beta}_{i, j}:=\frac{\delta_{i, j}}{\widehat{\delta}_{i, j}} .
$$

A general one-step finite difference scheme can be written as follows.

$$
\begin{aligned}
& \left(L_{\mathcal{A}}^{(2)} \eta\right)_{i, j}=F\left(t_{i, j}, \eta_{i, j},\left(L_{\mathcal{A}}^{(1)} \eta\right)_{i, j}, \chi^{\mathbf{f}}[\eta]_{i, j}, \chi^{\mathbf{v}}[\eta]_{i, j}\right), \quad(i, j) \in \mathcal{B}, \\
& \eta_{0,0}=r_{1}, \quad \eta_{n, 0}=r_{2},
\end{aligned}
$$

where

$$
\begin{aligned}
\left(L_{\mathcal{A}}^{(2)} \eta\right)_{i, j} & :=\frac{\widehat{\alpha}_{i, j} \eta_{i, j+1}-2 \eta_{i, j}+\widehat{\beta}_{i, j} \eta_{i, j-1}}{\widehat{\alpha}_{i, j} \widehat{\beta}_{i, j} \widehat{\delta}_{i, j}^{2}}, \quad\left(L_{\mathcal{A}}^{(1)} \eta\right)_{i, j}:=\frac{\eta_{i, j+1}-\eta_{i, j}}{\delta_{i, j}}, \\
\chi^{\mathbf{f}}[\eta]_{i, j}:= & \sum_{(l, v) \in \mathcal{T}} \delta_{l, v} K_{\mathbf{f}}\left(t_{i, j}, t_{l, v}, \eta_{l, v},\left(L_{\mathcal{A}}^{(1)} \eta\right)_{l, v},\left(L_{\mathcal{A}}^{(2)} \eta\right)_{l, v}\right), \\
\chi^{\mathbf{v}}[\eta]_{i, j}: & =\sum_{(l, v) \in \Delta_{i, j}} \delta_{l, v} K_{\mathbf{v}}\left(t_{i, j}, t_{l, v}, \eta_{l, v},\left(L_{\mathcal{A}}^{(1)} \eta\right)_{l, v},\left(L_{\mathcal{A}}^{(2)} \eta\right)_{l, v}\right) .
\end{aligned}
$$


Theorem 3. Let $f$ be a sufficiently smooth function on the interval $[a, b]$. Then we have

$$
\left|\chi^{l}[f]_{i, j}-z_{l}[f]\left(t_{i, j}\right)\right|=\mathcal{O}(h), \quad l=\mathbf{f}, \mathbf{v} .
$$

Proof. We can find

$$
\begin{aligned}
& \mid K_{\mathbf{f}}\left(t_{i, j}, t_{l, v}, f\left(t_{l, v}\right), f^{\prime}\left(t_{l, v}\right), f^{\prime \prime}\left(t_{l, v}\right)\right) \\
& \quad-K_{\mathbf{f}}\left(t_{i, j}, t_{l, v}, f\left(t_{l, v}\right),\left(L_{\mathcal{A}}^{(1)} f\right)_{l, v},\left(L_{\mathcal{A}}^{(2)} f\right)_{l, v}\right) \mid=\mathcal{O}(h) .
\end{aligned}
$$

Also we get

$$
\begin{gathered}
K_{\mathbf{f}}\left(t_{i, j}, s, f(s), f^{\prime}(s), f^{\prime \prime}(s)\right)-K_{\mathbf{f}}\left(t_{i, j}, t_{l, v}, f\left(t_{l, v}\right), f^{\prime}\left(t_{l, v}\right), f^{\prime \prime}\left(t_{l, v}\right)\right) \\
=\left(s-t_{l, v}\right) \frac{\partial K_{\mathbf{f}}}{\partial s}\left(t_{i, j}, \zeta_{s}^{l, v}, f\left(\zeta_{s}^{l, v}\right), f^{\prime}\left(\zeta_{s}^{l, v}\right), f^{\prime \prime}\left(\zeta_{s}^{l, v}\right)\right),
\end{gathered}
$$

where $\zeta_{s}^{l, v} \in\left[t_{l, v}, t_{l, v+1}\right]$. Therefore by using (2.8) and (2.9)we get

$$
\begin{aligned}
\int_{t_{l, v}}^{t_{l, v+1}} & K_{\mathbf{f}}\left(t_{i, j}, s, f(s), f^{\prime}(s), f^{\prime \prime}(s)\right) d s \\
& -\delta_{l, v} K_{\mathbf{f}}\left(t_{i, j}, t_{l, v}, f\left(t_{l, v}\right),\left(L_{\mathcal{A}}^{(1)} f\right)_{l, v},\left(L_{\mathcal{A}}^{(2)} f\right)_{l, v}\right) \\
= & \int_{t_{l, v}}^{t_{l, v+1}} K_{\mathbf{f}}\left(t_{i, j}, s, f(s), f^{\prime}(s), f^{\prime \prime}(s)\right) d s \\
& -\delta_{l, v} K_{\mathbf{f}}\left(t_{i, j}, t_{l, v}, f\left(t_{l, v}\right), f^{\prime}\left(t_{l, v}\right), f^{\prime \prime}\left(t_{l, v}\right)\right)+\mathcal{O}\left(h^{2}\right) \\
= & \frac{\delta_{l, v}^{2}}{2} \frac{\partial K_{\mathbf{f}}}{\partial s}\left(t_{i, j}, \zeta_{s}^{l, v}, f\left(\zeta_{s}^{l, v}\right), f^{\prime}\left(\zeta_{s}^{l, v}\right), f^{\prime \prime}\left(\zeta_{s}^{l, v}\right)\right)+\mathcal{O}\left(h^{2}\right) \\
\leq & \frac{h^{2}}{2} \frac{\partial K_{\mathbf{f}}}{\partial s}\left(t_{i, j}, \zeta_{s}^{l, v}, f\left(\zeta_{s}^{l, v}\right), f^{\prime}\left(\zeta_{s}^{l, v}\right), f^{\prime \prime}\left(\zeta_{s}^{l, v}\right)\right)+\mathcal{O}\left(h^{2}\right),
\end{aligned}
$$

then we can obtain

$$
\begin{aligned}
& \sum_{(l, v) \in \mathcal{T}}\left(\int_{t_{l, v}}^{t_{l, v+1}} K_{\mathbf{f}}\left(t_{i, j}, s, f(s), f^{\prime}(s), f^{\prime \prime}(s)\right) d s\right. \\
& \left.\quad-\delta_{l, v} K_{\mathbf{f}}\left(t_{i, j}, t_{l, v},, f\left(t_{l, v}\right),\left(L_{\mathcal{A}}^{(1)} f\right)_{l, v},\left(L_{\mathcal{A}}^{(2)} f\right)_{l, v}\right)\right) \\
& \leq \sum_{(l, v) \in \mathcal{T}} \frac{h^{2}}{2} \frac{\partial K_{\mathbf{f}}}{\partial s}\left(t_{i, j}, \zeta_{s}^{l, v}, f\left(\zeta_{s}^{l, v}\right), f^{\prime}\left(\zeta_{s}^{l, v}\right), f^{\prime \prime}\left(\zeta_{s}^{l, v}\right)\right)+\mathcal{O}(h) \\
& \leq n(m+1) \frac{h^{2}}{2} \max _{s \in[a, b]} \frac{\partial K_{\mathbf{f}}}{\partial s}\left(t_{i, j}, s, f(s), f^{\prime}(s), f^{\prime \prime}(s)\right)+\mathcal{O}(h) \\
& \leq \frac{(b-a)(m+1) h}{2 h^{\prime}} h \max _{s \in[a, b]} \frac{\partial K_{\mathbf{f}}}{\partial s}\left(t_{i, j}, s, f(s), f^{\prime}(s), f^{\prime \prime}(s)\right)+\mathcal{O}(h)=\mathcal{O}(h),
\end{aligned}
$$

then by using (2.10), we can say that

$$
\chi^{\mathbf{f}}[f]_{i, j}-z_{\mathbf{f}}[f]\left(t_{i, j}\right)=\mathcal{O}(h) .
$$

Similarly, we can find (2.7) for linear case and $l=\mathbf{v}$. 
Definition 3. For any function $u$, we define

$$
\mathcal{R}(u):=\left\{u\left(t_{i, j}\right) ; \quad(i, j) \in \mathcal{A}\right\},
$$

also we define

$$
\eta:=\left\{\eta_{i, j} ;(i, j) \in \mathcal{A}\right\}, \quad L_{\mathcal{A}}^{(l)} \eta:=\left\{\left(L_{\mathcal{A}}^{(l)} \eta\right)_{i, j} ;(i, j) \in \mathcal{A}\right\}, \quad l=1,2 .
$$

For the above finite difference scheme we have the following estimate

$$
\|\eta-\mathcal{R}(y)\|_{\infty}=\mathcal{O}(h), \quad\left\|L_{\mathcal{A}}^{(l)} \eta-\mathcal{R}\left(y^{(l)}\right)\right\|_{\infty}=\mathcal{O}\left(h^{l}\right), \quad l=1,2,
$$

where $\eta$ and $L_{\mathcal{A}}^{(l)} \eta$ is defined in the Definition 3 .

\subsection{Deviation of the error estimation}

We study the deviation of the error estimation for (1.1)-(1.2). We consider the Dirichlet problem

$$
y^{\prime \prime}(t)=f(t), a \leq t \leq b, \quad y(a)=y(b)=0,
$$

where $f(t)$ is permitted to have jump discontinuities in the points belonging to $Z_{n}$. For the discretization form of (2.11), i.e.,

$$
\begin{aligned}
& \left(L_{\mathcal{A}}^{(2)} \eta\right)_{i, j}=f\left(t_{i, j}\right), \quad(i, j) \in \mathcal{B}, \\
& \eta_{0,0}=0, \quad \eta_{n, 0}=0
\end{aligned}
$$

according to $[1,3]$, we have the following lemmas.

Lemma 3. The unique solution $\eta$ of (2.12)-(2.13) is given by

$$
\eta_{i, j}=\sum_{(l, v) \in \mathcal{B}} \widehat{\delta}_{l, v} G\left(t_{i, j}, t_{l, v}\right) f\left(t_{l, v}\right),
$$

where $G(t, \tau)$ is Green's function

$$
G(t, \tau)= \begin{cases}(b-t)(a-\tau) / b-a, & a \leq \tau \leq t \leq b \\ (b-\tau)(a-t) / b-a, & a \leq t \leq \tau \leq b .\end{cases}
$$

Lemma 4. For $v \in \widehat{\mathcal{C}}_{2}\left[t_{i, j-1}, t_{i, j}, t_{i, j+1}\right]$, where

$$
\begin{aligned}
& \widehat{\mathcal{C}}_{2}\left[t_{i, j-1}, t_{i, j}, t_{i, j+1}\right]:=\left\{v \in \mathcal{C}^{1}\left[t_{i, j-1}, t_{i, j+1}\right]: v^{\prime \prime}\right. \text { continuous on } \\
& \left.\left[t_{i, j-1}, t_{i, j}\right) \cup\left(t_{i, j}, t_{i, j+1}\right], \lim _{t \uparrow t_{i, j}} v^{\prime \prime} \in \mathbb{R}, \lim _{t \downarrow t_{i, j}} v^{\prime \prime} \in \mathbb{R} \text { exist }\right\},
\end{aligned}
$$

we have

$$
\left(L_{\mathcal{A}}^{(2)} v\right)_{i, j}=\int_{-\widehat{\alpha}_{i, j}}^{\widehat{\beta}_{i, j}} R_{i, j}(\xi) v^{\prime \prime}\left(t_{i, j}+\widehat{\delta}_{i, j} \xi\right) d \xi
$$

with kernel

$$
R_{i, j}(\xi)= \begin{cases}1+\xi / \widehat{\alpha}_{i, j}, & \xi \in\left[-\widehat{\alpha}_{i, j}, 0\right] \\ 1-\xi / \widehat{\beta}_{i, j}, & \xi \in\left[0, \widehat{\beta}_{i, j}\right]\end{cases}
$$


As $[1,3]$ we can find "the exact finite difference" for (1.1) as follows

$$
\left(L_{\mathcal{A}}^{(2)} p\right)_{i, j}=\mathcal{I}_{\mathcal{A}}\left(F\left(t_{i, j}, p\left(t_{i, j}\right), p^{\prime}\left(t_{i, j}\right), z_{\mathbf{f}}[p]\left(t_{i, j}\right), z_{\mathbf{v}}[p]\left(t_{i, j}\right)\right)\right)
$$

where

$$
\mathcal{I}_{\mathcal{A}}\left(w\left(t_{i, j}\right)\right):=\int_{-\widehat{\alpha}_{i, j}}^{\widehat{\beta}_{i, j}} R_{i, j}(\xi) w\left(t_{i, j}+\widehat{\delta}_{i, j} \xi\right) d \xi
$$

We can say that a solution of problem (1.1)-(1.2) satisfies in the exact finite difference scheme. Since according to the collocation method, we can say that

$$
p^{\prime \prime}\left(t_{i, j}\right)-F\left(t_{i, j}, p\left(t_{i, j}\right), p^{\prime}\left(t_{i, j}\right), z_{\mathbf{f}}[p]\left(t_{i, j}\right), z_{\mathbf{v}}[p]\left(t_{i, j}\right)\right) \equiv 0,(i, j) \in X(n),
$$

therefore we define the defect at $t_{i, j}$ as follows

$$
D_{i, j}:=\left(L_{\mathcal{A}}^{(2)} p\right)_{i, j}-\mathcal{I}_{\mathcal{A}}\left(F\left(t_{i, j}, p\left(t_{i, j}\right), p^{\prime}\left(t_{i, j}\right), z_{\mathbf{f}}[p]\left(t_{i, j}\right), z_{\mathbf{v}}[p]\left(t_{i, j}\right)\right)\right),(i, j) \in \mathcal{B} .
$$

In order to compute the integral in this expression, we use a quadrature formula. When $t_{i, j} \in X(n)$ we have $[1,3]$

$$
\begin{gathered}
\mathcal{I}_{\mathcal{A}}\left(F\left(\cdot, p, p^{\prime}, z_{\mathbf{f}}[p], z_{\mathbf{v}}[p]\right), t_{i, j}\right) \approx Q_{\mathcal{A}}\left(F\left(t_{i, j}, p\left(t_{i, j}\right), p^{\prime}\left(t_{i, j}\right), \widetilde{z}_{\mathbf{f}}[p]\left(t_{i, j}\right), \widetilde{z}_{\mathbf{v}}[p]\left(t_{i, j}\right)\right)\right) \\
:=\sum_{k=0}^{m+1} \gamma_{i, j}^{k} F\left(t_{i, k}, p\left(t_{i, k}\right), p^{\prime}\left(t_{i, k}\right), \widetilde{z}_{\mathbf{f}}[p]\left(t_{i, k}\right), \widetilde{z}_{\mathbf{v}}[p]\left(t_{i, k}\right)\right)
\end{gathered}
$$

where $\gamma_{i, j}^{k}=\int_{-\widehat{\alpha}_{i, j}}^{\widehat{\beta}_{i, j}} R_{i, j}(\xi) L_{k}\left(\rho_{j}+\xi \frac{\widehat{\delta}_{i, j}}{h_{i}}\right) d \xi$. Also for $t_{i, 0}=\tau_{i}$ we have $[1,3]$

$$
\begin{aligned}
\mathcal{I}_{\mathcal{A}}( & F\left(t_{i, 0}, p\left(t_{i, 0}\right), p^{\prime}\left(t_{i, 0}\right), z_{\mathbf{f}}[p]\left(t_{i, 0}\right), z_{\mathbf{v}}[p]\left(t_{i, 0}\right)\right) \\
\approx & Q_{\mathcal{A}}\left(F\left(t_{i, 0}, p\left(t_{i, 0}\right), p^{\prime}\left(t_{i, 0}\right), \widetilde{z}_{\mathbf{f}}[p]\left(t_{i, 0}\right), \widetilde{z}_{\mathbf{v}}[p]\left(t_{i, 0}\right)\right)\right) \\
:= & \sum_{k=0}^{m+1} \gamma_{i, 0}^{k+} F\left(t_{i, k}, p\left(t_{i, k}\right), p^{\prime}\left(t_{i, k}\right), \widetilde{z}_{\mathbf{f}}[p]\left(t_{i, k}\right), \widetilde{z}_{\mathbf{v}}[p]\left(t_{i, k}\right)\right) \\
& +\sum_{k=0}^{m+1} \gamma_{i, 0}^{k-} F\left(t_{i-1, k}, p\left(t_{i-1, k}\right), p^{\prime}\left(t_{i-1, k}\right), \widetilde{z}_{\mathbf{f}}[p]\left(t_{i-1, k}\right), \widetilde{z}_{\mathbf{v}}[p]\left(t_{i-1, k}\right)\right),
\end{aligned}
$$

where

$$
\gamma_{i, 0}^{k+}=\int_{0}^{\widehat{\beta}_{i, 0}} R_{i, 0}(\xi) L_{k}\left(\xi \frac{\widehat{\delta}_{i, 0}}{h_{i}}\right) d \xi, \quad \gamma_{i, 0}^{k-}=\int_{-\widehat{\alpha}_{i, 0}}^{0} R_{i, 0}(\xi) L_{k}\left(1+\xi \frac{\widehat{\delta}_{i, 0}}{h_{i-1}}\right) d \xi .
$$

For the above quadrature formula we can find the following lemma.

Lemma 5. For sufficiently smooth $f$ the following error holds

$$
\mathcal{I}_{\mathcal{A}}\left(f, t_{i, j}\right)-Q_{\mathcal{A}}\left(f, t_{i, j}\right)=\mathcal{O}\left(h^{m+2}\right) .
$$

Also when $m$ is odd and the nodes $\rho_{i}$ are distributed symmetrically, we have the following relation

$$
\mathcal{I}_{\mathcal{A}}\left(f, t_{i, j}\right)-Q_{\mathcal{A}}\left(f, t_{i, j}\right)=\mathcal{O}\left(h^{m+3}\right) .
$$


Then we consider defect at $t_{i, j}$ as follows

$$
D_{i, j} \approx\left(L_{\mathcal{A}}^{(2)} p\right)_{i, j}-Q_{\mathcal{A}}\left(F\left(t_{i, j}, p\left(t_{i, j}\right), p^{\prime}\left(t_{i, j}\right), z_{\mathbf{f}}[p]\left(t_{i, j}\right), z_{\mathbf{v}}[p]\left(t_{i, j}\right)\right)\right),(i, j) \in \mathcal{B}
$$

At this step we define $\pi=\left\{\pi_{i, j} ;(i, j) \in \mathcal{A}\right\}$ as the solution of the following finite difference

$$
\begin{aligned}
& \left(L_{\mathcal{A}}^{(2)} \pi\right)_{i, j}=F\left(t_{i, j}, \pi_{i, j},\left(L_{\mathcal{A}}^{(1)} \pi\right)_{i, j}, \chi^{\mathbf{f}}[\pi]_{i, j}, \chi^{\mathbf{v}}[\pi]_{i, j}\right)+D_{i, j},(i, j) \in \mathcal{B}, \\
& \pi_{0,0}=r_{1}, \quad \pi_{n, 0}=r_{2} .
\end{aligned}
$$

We define $\mathbf{D}:=\left\{D_{i, j} ;(i, j) \in \mathcal{B}\right\}$. For small value $\mathbf{D}$, we have

$$
\pi-\mathcal{R}(p) \approx \eta-\mathcal{R}(y)
$$

We define $\varepsilon$ and $e$ as $\varepsilon:=\pi-\eta \approx \mathcal{R}(p)-\mathcal{R}(y)=$ : $e$. An estimate for the error $e$ can be found in Theorem 2. The deviation of the error can be written in the following form $\theta:=e-\varepsilon$. By using (2.14) and Lemma 5 for linear and nonlinear case we can easily prove the following lemmas.

Lemma 6. The defined defect in (2.14) has order $\mathcal{O}\left(h^{m}\right)$.

Lemma 7. The $\pi-\eta$ has order $\mathcal{O}\left(h^{m}\right)$.

\section{Analysis of the deviation of the error}

Lemma 8. For the linear $z_{l}[\cdot](t)(l=\mathbf{f}, \mathbf{v})$ we have

$$
\left|\chi^{l}[e]_{i, j}-\widetilde{z}_{l}[e]\left(t_{i, j}\right)\right|=\mathcal{O}\left(h^{m}\right), \quad l=\mathbf{f}, \mathbf{v} .
$$

Proof. When $l=\mathbf{f}$ by using Lemma 1 we can write

$$
\left|\chi^{\mathbf{f}}[e]_{i, j}-\widetilde{z}_{\mathbf{f}}[e]\left(t_{i, j}\right)\right| \leq\left|\chi^{\mathbf{f}}[e]_{i, j}-z_{\mathbf{f}}[e]\left(t_{i, j}\right)\right|+\underbrace{\left|z_{\mathbf{f}}[e]\left(t_{i, j}\right)-\widetilde{z}_{\mathbf{f}}[e]\left(t_{i, j}\right)\right|}_{\mathcal{O}\left(h^{m+2}\right)},
$$

also by using Theorem 2 and Theorem 3 we can write

$$
\begin{aligned}
& \left|\chi^{\mathbf{f}}[e]_{i, j}-z_{\mathbf{f}}[e]\left(t_{i, j}\right)\right|=\mid \sum_{(l, v) \in \mathcal{T}} \sum_{m=0}^{2}\left(\int_{t_{l, v}}^{t_{l, v+1}} \Lambda_{m, \mathbf{f}}\left(t_{i, j}, s\right) e^{(m)}(s) d s\right. \\
& \left.-\delta_{l, v} \Lambda_{m, \mathbf{f}}\left(t_{i, j}, t_{l, v}\right) e^{(m)}\left(t_{l, v}\right)\right) \mid+\mathcal{O}\left(h^{m}\right) \leq \frac{(b-a)(m+1) h}{2 h^{\prime}} h
\end{aligned}
$$

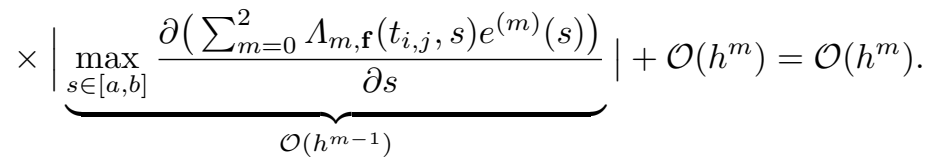

In a similar way to the $l=\mathbf{f}$, we can prove (3.1) for $l=\mathbf{v}$. 


\subsection{Linear case}

Theorem 4. Consider the SFVID equation (1.3) with boundary conditions (1.2). Assume that the SFVID problem has a unique and sufficiently smooth solution. Then the following estimate holds

$$
\|\theta\|_{\infty}=\|e-\varepsilon\|_{\infty}=\mathcal{O}\left(h^{m+1}\right),
$$

where $e$ is error, $\varepsilon$ is the error estimate and $\theta$ is the deviation of the error estimate.

Proof. Since $F$ and $z_{l}[\cdot](t)(l=\mathbf{f}, \mathbf{v})$ are linear then by using $(2.5),(2.14)$ and (2.15) we get

$$
\begin{aligned}
& \left(L_{\mathcal{A}}^{(2)} \theta\right)_{i, j}=a_{1}\left(t_{i, j}\right)\left(L_{\mathcal{A}}^{(1)} \theta\right)_{i, j}+a_{2}\left(t_{i, j}\right) \theta_{i, j}+\sum_{k=\mathbf{f}, \mathbf{v}} \chi^{k}[\theta]_{i, j} \\
& +\underbrace{\mathcal{I}_{\mathcal{A}}\left(a_{1} e^{\prime}+a_{2} e, t_{i, j}\right)-\left(a_{1}\left(t_{i, j}\right)\left(L_{\mathcal{A}}^{(1)} e\right)_{i, j}+a_{2}\left(t_{i, j}\right) e\left(t_{i, j}\right)\right)}_{I_{4}} \\
& +\underbrace{\left(\mathcal{Q}_{\mathcal{A}}-\mathcal{I}_{\mathcal{A}}\right)\left(a_{1} p^{\prime}+a_{2} p+a_{3}, t_{i, j}\right)}_{I 5}+\underbrace{\sum_{k=\mathbf{f}, \mathbf{v}}\left(\mathcal{I}_{A}\left(z_{k}[e], t_{i, j}\right)-\chi^{k}[e]_{i, j}\right)}_{I_{6}} \\
& +\underbrace{\sum_{k=\mathbf{f}, \mathbf{v}}\left(Q_{A}\left(\widetilde{z}_{k}[p]\left(t_{i, j}\right)\right)-\mathcal{I}_{A}\left(z_{k}[p], t_{i, j}\right)\right)}_{I_{7}},
\end{aligned}
$$

by using Lemma 6.1 in [1] and [3] we can say

$$
I_{4}=\left(g_{i, j}-\dot{g}_{i, j}-\ddot{g}_{i, j}\right)+\frac{1}{2 \widehat{\delta}_{i, j}}\left(\delta_{i, j} \phi_{i, j+\frac{1}{2}}-\delta_{i, j-1} \phi_{i, j-\frac{1}{2}}\right)+\mathcal{O}\left(h^{m+2}\right),
$$

where

$$
\begin{aligned}
& g_{i, j}:=\int_{-\widehat{\alpha}_{i, j}}^{0}\left(\frac{\xi}{\widehat{\alpha}_{i, j}}+1\right) a_{1}\left(t_{i, j}+\xi \widehat{\delta}_{i, j}\right) e^{\prime}\left(t_{i, j}+\xi \widehat{\delta}_{i, j}\right) d \xi \\
& \dot{g}_{i, j}:=\int_{0}^{\widehat{\beta}_{i, j}}\left(\frac{\xi}{\widehat{\beta}_{i, j}}-1\right) a_{1}\left(t_{i, j}+\xi \widehat{\delta}_{i, j}\right) e^{\prime}\left(t_{i, j}+\xi \widehat{\delta}_{i, j}\right) d \xi \\
& \ddot{g}_{i, j}:=\frac{a_{1}\left(t_{i, j}\right)}{\widehat{\beta}_{i, j}} \int_{0}^{\widehat{\beta}_{i, j}} e^{\prime}\left(t_{i, j}+\xi \widehat{\delta}_{i, j}\right) d \xi \\
& \phi_{i, j+\frac{1}{2}}:=\delta_{i, j} \int_{-\frac{1}{2}}^{\frac{1}{2}}\left(u^{2}+\frac{1}{4}\right) Q\left(t_{i, j+\frac{1}{2}}+\delta_{i, j} u\right) d u \\
& \phi_{i, j-\frac{1}{2}}:=\delta_{i, j-1} \int_{-\frac{1}{2}}^{\frac{1}{2}}\left(u^{2}+\frac{1}{4}\right) Q\left(t_{i, j-\frac{1}{2}}+\delta_{i, j-1} u\right) d u
\end{aligned}
$$

with $Q(t)=\left(a_{2}(t) e(t)\right)^{\prime}$. We can easily prove that

$$
g_{i, j}=\mathcal{O}\left(h^{m+1}\right), \quad \dot{g}_{i, j}=\mathcal{O}\left(h^{m+1}\right), \quad \ddot{g}_{i, j}=\mathcal{O}\left(h^{m}\right), \quad \phi_{i, j \pm \frac{1}{2}}=\mathcal{O}\left(h^{m+1}\right) .
$$


Also according to Theorem 6.1 in [1] and [3], we can obtain $I_{5}=\mathcal{O}\left(h^{m+2}\right)$. Also since $p \in \Pi_{m+1}$ and $\Lambda_{l, k}(t, s) \quad(l=0,1,2 \& k=\mathbf{f}, \mathbf{v})$ are sufficiently smooth then by using lemma 5 we can say that $I_{7}=\mathcal{O}\left(h^{m+2}\right)$.

In a similar way to the $I_{4}$, we can find $I_{6}=\Upsilon_{\mathbf{f}}\left(t_{i, j}\right)+\Upsilon_{\mathbf{v}}\left(t_{i, j}\right)$,

$$
\begin{aligned}
& \Upsilon_{\mathbf{f}}\left(t_{i, j}\right):=\sum_{(w, v) \in \mathcal{T}}\left(\dot{\Omega}_{\mathbf{f}}^{w, v}\left(t_{i, j}\right)-\ddot{\Omega}_{\mathbf{f}}^{w, v}\left(t_{i, j}\right)\right), \\
& \Upsilon_{\mathbf{v}}\left(t_{i, j}\right):=\sum_{(w, v) \in \Delta_{i, j}}\left(\dot{\Omega}_{\mathbf{v}}^{w, v}\left(t_{i, j}\right)-\ddot{\Omega}_{\mathbf{v}}^{w, v}\left(t_{i, j}\right)\right) \\
&+\sum_{l=0}^{2} \int_{-\widehat{\alpha}_{i, j}}^{\widehat{\beta}_{i, j}} \int_{t_{i, j}}^{t_{i, j}+\xi \widehat{\delta}_{i, j}} \Lambda_{l, v}\left(t_{i, j}+\xi \widehat{\delta}_{i, j}, s\right) e^{(l)}(s) d s d \xi,
\end{aligned}
$$

where

$$
\begin{aligned}
& \dot{\Omega}_{k}^{w, v}\left(t_{i, j}\right):=\Psi_{k}^{w, v}\left(t_{i, j}\right)-\breve{\Psi}_{k}^{w, v}\left(t_{i, j}\right), \quad k=\mathbf{f}, \mathbf{v} \\
& \ddot{\Omega}_{k}^{w, v}\left(t_{i, j}\right):=\bar{\Psi}_{k}^{w, v}\left(t_{i, j}\right)-\breve{\Psi}_{k}^{w, v}\left(t_{i, j}\right), \quad k=\mathbf{f}, \mathbf{v}
\end{aligned}
$$

with

$$
\begin{aligned}
& \Psi_{k}^{w, v}\left(t_{i, j}\right):= \sum_{l=0}^{2} \int_{t_{w, v}}^{t_{w, v+1}} \int_{-\widehat{\alpha}_{i, j}}^{0}\left(\frac{\xi}{\widehat{\alpha}_{i, j}}+1\right) \Lambda_{l, k}\left(t_{i, j}+\xi \widehat{\delta}_{i, j}, s\right) e^{(l)}(s) d \xi d s \\
& \bar{\Psi}_{k}^{w, v}\left(t_{i, j}\right):=\sum_{l=0}^{2} \int_{t_{w, v}}^{t_{w, v+1}} \int_{0}^{\widehat{\beta}_{i, j}}\left(\frac{\xi}{\widehat{\beta}_{i, j}}-1\right) \Lambda_{l, k}\left(t_{i, j}+\xi \widehat{\delta}_{i, j}, s\right) e^{(l)}(s) d \xi d s \\
& \breve{\Psi}_{k}^{w, v}\left(t_{i, j}\right):= \\
& \quad \int_{-\widehat{\alpha}_{i, j}}^{0} \frac{1}{2} \delta_{w, v}\left(\Lambda_{0, k}\left(t_{i, j}, t_{w, v}\right) e\left(t_{w, v}\right)\right. \\
&\left.+\sum_{l=1}^{2} \Lambda_{l, k}\left(t_{i, j}, t_{w, v}\right)\left(L_{\mathcal{A}}^{(l)} e\right)_{w, v}\right) d \xi \\
& \breve{\Psi}_{k}^{w, v}\left(t_{i, j}\right):=-\int_{0}^{\widehat{\beta}_{i, j}} \frac{1}{2} \delta_{w, v}\left(\Lambda_{0, k}\left(t_{i, j}, t_{w, v}\right) e\left(t_{w, v}\right)\right. \\
&\left.+\sum_{l=1}^{2} \Lambda_{l, k}\left(t_{i, j}, t_{w, v}\right)\left(L_{\mathcal{A}}^{(l)} e\right)_{w, v}\right) d \xi .
\end{aligned}
$$

By using Theorem 2, we obtain

$$
\Upsilon_{k}\left(t_{i, j}\right)=\mathcal{O}\left(h^{m}\right), \quad k=\mathbf{f}, \mathbf{v} .
$$

Therefore we can rewrite (3.2) as follows

$$
\begin{aligned}
\left(L_{\mathcal{A}}^{(2)} \theta\right)_{i, j} & =a_{1}\left(t_{i, j}\right)\left(L_{\mathcal{A}}^{(1)} \theta\right)_{i, j}+a_{2}\left(t_{i, j}\right) \theta_{i, j}+\sum_{k=\mathbf{f}, \mathbf{v}} \chi^{k}[\theta]_{i, j} \\
& +\left(g_{i, j}-\dot{g}_{i, j}-\ddot{g}_{i, j}\right)+\frac{1}{2 \widehat{\delta}_{i, j}}\left(\delta_{i, j} \phi_{i, j+\frac{1}{2}}-\delta_{i, j-1} \phi_{i, j-\frac{1}{2}}\right) \\
& +\Upsilon_{\mathbf{f}}\left(t_{i, j}\right)+\Upsilon_{\mathbf{v}}\left(t_{i, j}\right)+\mathcal{O}\left(h^{m+2}\right) .
\end{aligned}
$$


In this step we define

$$
\begin{aligned}
& H:=\left\{g_{i, j}-\dot{g}_{i, j}-\ddot{g}_{i, j} ;(i, j) \in \mathcal{B}\right\}, \Theta=\left\{\Upsilon_{\mathbf{f}}\left(t_{i, j}\right)+\Upsilon_{\mathbf{v}}\left(t_{i, j}\right) ;(i, j) \in \mathcal{B}\right\}, \\
& \Phi:=\left\{\delta_{i, j} \phi_{i, j+\frac{1}{2}}-\delta_{i, j-1} \phi_{i, j-\frac{1}{2}} ;(i, j) \in \mathcal{B}\right\} .
\end{aligned}
$$

Then by using Lemma 3 we find

$$
\theta_{i, j}=\left(\left(L_{\mathcal{A}}^{2}\right)^{-1} H\right)_{i, j}+\frac{1}{2 \widehat{\delta}_{i, j}}\left(\left(L_{\mathcal{A}}^{2}\right)^{-1} \Phi\right)_{i, j}+\left(\left(L_{\mathcal{A}}^{2}\right)^{-1} \Theta\right)_{i, j} .
$$

Let

$$
\widehat{g}=\max _{i, j}|H|=\mathcal{O}\left(h^{m}\right), \quad \widehat{\phi}=\max _{i, j}|\Phi|=\mathcal{O}\left(h^{m+1}\right), \quad \widehat{\Upsilon}=\max _{i, j}|\Theta|=\mathcal{O}\left(h^{m}\right),
$$

therefore we get

$$
\begin{aligned}
\|\theta\|_{\infty} & \leq h\left(\max _{w, x} \sum_{(i, j) \in \mathcal{B}} G\left(t_{w, x}, t_{i, j}\right) \widehat{g}\right)+\frac{1}{2}\left(\max _{w, x} \sum_{(i, j) \in \mathcal{B}} G\left(t_{w, x}, t_{i, j}\right) \widehat{\phi}\right) \\
& +h\left(\max _{w, x} \sum_{(i, j) \in \mathcal{B}} G\left(t_{w, x}, t_{i, j}\right) \widehat{\Upsilon}\right)=\mathcal{O}\left(h^{m+1}\right) .
\end{aligned}
$$

\subsection{Nonlinear case}

Definition 4. For nonlinear and linear $z_{l}[\cdot](l=\mathbf{f}, \mathbf{v})$ we define

$$
\begin{aligned}
\bar{\chi}^{\mathbf{f}}[\varepsilon]_{i, j} & :=\sum_{(l, v) \in \mathcal{T}} \delta_{l, v}\left(\Gamma_{0, \mathbf{f}}^{\varepsilon}\left(t_{i, j}, t_{l, v}\right) \varepsilon_{l, v}+\sum_{m=1}^{2} \Gamma_{m, \mathbf{f}}^{\varepsilon}\left(t_{i, j}, t_{l, v}\right)\left(L_{\mathcal{A}}^{(m)} \varepsilon\right)_{l, v}\right), \\
\bar{\chi}^{\mathbf{v}}[\varepsilon]_{i, j} & :=\sum_{(l, v) \in \Delta_{i, j}} \delta_{l, v}\left(\Gamma_{0, \mathbf{v}}^{\varepsilon}\left(t_{i, j}, t_{l, v}\right) \varepsilon_{l, v}+\sum_{m=1}^{2} \Gamma_{m, \mathbf{v}}^{\varepsilon}\left(t_{i, j}, t_{l, v}\right)\left(L_{\mathcal{A}}^{(m)} \varepsilon\right)_{l, v}\right),
\end{aligned}
$$

where, for linear case

$$
\Gamma_{m, k}^{\varepsilon}\left(t_{i, j}, t_{l, v}\right):=\Lambda_{m, k}\left(t_{i, j}, t_{l, v}\right), \quad m=0,1,2, \quad k=\mathbf{f}, \mathbf{v}
$$

and for nonlinear case and $k=\mathbf{f}, \mathbf{v}$ we define

$$
\begin{aligned}
& \Gamma_{m, k}^{\varepsilon}\left(t_{i, j}, t_{l, v}\right):= \\
& \begin{cases}\int_{0}^{1}\left(K_{k}\right)_{u}\left(t_{i, j}, t_{l, v}, \eta_{l, v}+\tau \varepsilon_{l, v},\left(L_{\mathcal{A}}^{(1)} \pi\right)_{l, v},\left(L_{\mathcal{A}}^{(2)} \pi\right)_{l, v}\right) d \tau, & m=0, \\
\int_{0}^{1}\left(K_{k}\right)_{u^{\prime}}\left(t_{i, j}, t_{l, v}, \eta_{l, v},\left(L_{\mathcal{A}}^{(1)} \eta\right)_{l, v}+\tau\left(L_{\mathcal{A}}^{(1)} \varepsilon\right)_{l, v},\left(L_{\mathcal{A}}^{(2)} \pi\right)_{l, v}\right) d \tau, & m=1, \\
\int_{0}^{1}\left(K_{k}\right)_{u^{\prime \prime}}\left(t_{i, j}, t_{l, v}, \eta_{l, v},\left(L_{\mathcal{A}}^{(1)} \eta\right)_{l, v},\left(L_{\mathcal{A}}^{(2)} \eta\right)_{l, v}+\tau\left(L_{\mathcal{A}}^{(2)} \varepsilon\right)_{l, v}\right) d \tau, & m=2 .\end{cases}
\end{aligned}
$$

Definition 5. For nonlinear and linear $z_{l}[\cdot](t)(l=\mathbf{f}, \mathbf{v})$ we define

$$
\begin{aligned}
& \bar{z}_{\mathbf{f}}[e]\left(t_{i, j}\right):=\sum_{m=0}^{2} \int_{a}^{b} \Gamma_{m, \mathbf{f}}^{e}\left(t_{i, j}, s\right) e^{(m)}(s) d s, \\
& \bar{z}_{\mathbf{v}}[e]\left(t_{i, j}\right):=\sum_{m=0}^{2} \int_{a}^{t_{i, j}} \Gamma_{m, \mathbf{v}}^{e}\left(t_{i, j}, s\right) e^{(m)}(s) d s,
\end{aligned}
$$


where, for linear case we define

$$
\Gamma_{m, k}^{e}\left(t_{i, j}, s\right):=\Lambda_{m, k}\left(t_{i, j}, s\right), \quad m=0,1,2, \quad k=\mathbf{f}, \mathbf{v}
$$

and for nonlinear case and $k=\mathbf{f}, \mathbf{v}$ we define

$$
\begin{aligned}
& \Gamma_{m, k}^{e}\left(t_{i, j}, s\right):= \\
& \begin{cases}\int_{0}^{1}\left(K_{k}\right)_{u}\left(t_{i, j}, s, y(s)+\tau e(s), p^{\prime}(s), p^{\prime \prime}(s)\right) d \tau, & m=0, \\
\int_{0}^{1}\left(K_{k}\right)_{u^{\prime}}\left(t_{i, j}, s, y(s), y^{\prime}(s)+\tau e^{\prime}(s), p^{\prime \prime}(s)\right) d \tau, & m=1, \\
\int_{0}^{1}\left(K_{k}\right)_{u^{\prime \prime}}\left(t_{i, j}, s, y(s), y^{\prime}(s), y^{\prime \prime}(s)+\tau e^{\prime \prime}(s)\right) d \tau, & m=2 .\end{cases}
\end{aligned}
$$

Now we can easily find the following lemma.

Lemma 9. For linear and nonlinear $z_{l}[\cdot](t)(l=\mathbf{f}, \mathbf{v})$, we get

$$
\begin{aligned}
& \chi^{k}[\pi]_{i, j}-\chi^{k}[\eta]_{i, j}=\bar{\chi}^{k}[\varepsilon]_{i, j}, \quad k=\mathbf{f}, \mathbf{v}, \\
& z_{k}[p]\left(t_{i, j}\right)-z_{k}[y]\left(t_{i, j}\right)=\bar{z}_{k}[e]\left(t_{i, j}\right), \quad k=\mathbf{f}, \mathbf{v} .
\end{aligned}
$$

Lemma 10. For linear and nonlinear $z_{l}[\cdot](t)(l=\mathbf{f}, \mathbf{v})$, we have

$$
\left|\bar{\chi}^{k}[\varepsilon]_{i, j}-\bar{z}_{k}[e]\left(t_{i, j}\right)\right|=\mathcal{O}\left(h^{m}\right), \quad k=\mathbf{f}, \mathbf{v} .
$$

Proof. In the linear case by using Lemma 7, Theorem 2 and the Integral mean value theorem we get

$$
\begin{aligned}
\bar{\chi}^{\mathbf{f}}[\varepsilon]_{i, j}- & \bar{z}_{\mathbf{f}}[e]\left(t_{i, j}\right)=\chi^{\mathbf{f}}[\varepsilon]_{i, j}-z_{\mathbf{f}}[e]\left(t_{i, j}\right) \\
= & \sum_{(l, v) \in \mathcal{T}} \delta_{l, v}\left(\Lambda_{0, \mathbf{f}}\left(t_{i, j}, t_{l, v}\right) \varepsilon_{l, v}+\sum_{m=1}^{2} \Lambda_{m, \mathbf{f}}\left(t_{i, j}, t_{l, v}\right)\left(L_{\mathcal{A}}^{(m)} \varepsilon\right)_{l, v}\right) \\
& -\sum_{l=0}^{2} \int_{a}^{b} \Lambda_{l, \mathbf{f}}\left(t_{i, j}, s\right) e^{(l)}(s) d s \\
\leq & \frac{(b-a)(m+1) h}{h^{\prime}} \mathcal{O}\left(h^{m}\right) \sum_{l=0}^{2} \max _{(l, v) \in \mathcal{A}} \Lambda_{l, \mathbf{f}}\left(t_{i, j}, t_{l, v}\right) \\
& +\mathcal{O}\left(h^{m}\right)(b-a) \sum_{l=0}^{2} \Lambda_{l, \mathbf{f}}\left(t_{i, j}, \zeta_{i, j}^{l}\right)=\mathcal{O}\left(h^{m}\right)
\end{aligned}
$$

where $\zeta_{i, j}^{l} \in(a, b)$. In this step we study nonlinear case. According to (3.3) and (3.4) we obtain

$$
\begin{aligned}
\left|\bar{\chi}^{\mathbf{f}}[\varepsilon]_{i, j}\right|=\left|\chi^{\mathbf{f}}[\pi]_{i, j}-\chi^{\mathbf{f}}[\eta]_{i, j}\right| & =\sum_{(l, v) \in \mathcal{T}} \delta_{l, v}\left(K_{\mathbf{f}}\left(t_{i, j}, t_{l, v}, \pi_{l, v},\left(L_{\mathcal{A}}^{(1)} \pi\right)_{l, v},\left(L_{\mathcal{A}}^{(2)} \pi\right)_{l, v}\right)\right. \\
& \left.-K_{\mathbf{f}}\left(t_{i, j}, t_{l, v}, \eta_{l, v},\left(L_{\mathcal{A}}^{(1)} \eta\right)_{l, v},\left(L_{\mathcal{A}}^{(2)} \eta\right)_{l, v}\right)\right) \leq \sum_{(l, v) \in \mathcal{T}} \delta_{l, v}\left(C\left|\pi_{l, v}-\eta_{l, v}\right|\right. \\
& \left.+C\left|\left(L_{\mathcal{A}}^{(1)} \pi\right)_{l, v}-\left(L_{\mathcal{A}}^{(1)} \eta\right)_{l, v}\right|+C\left|\left(L_{\mathcal{A}}^{(2)} \pi\right)_{l, v}-\left(L_{\mathcal{A}}^{(2)} \eta\right)_{l, v}\right|\right) \\
& \leq C \mathcal{O}\left(h^{m}\right) h n(m+1) \leq C \frac{(b-a)(m+1) h}{h^{\prime}} \mathcal{O}\left(h^{m}\right)=\mathcal{O}\left(h^{m}\right),
\end{aligned}
$$


also

$$
\begin{aligned}
\left|\bar{z}_{\mathbf{f}}[e]\left(t_{i, j}\right)\right| & =\left|z_{\mathbf{f}}[p]\left(t_{i, j}\right)-z_{\mathbf{f}}[y]\left(t_{i, j}\right)\right| \\
& \leq \int_{a}^{b}\left|K_{\mathbf{f}}\left(t_{i, j}, s, p(s), p^{\prime}(s), p^{\prime \prime}(s)\right)-K_{\mathbf{f}}\left(t_{i, j}, s, y(s), y^{\prime}(s), y^{\prime \prime}(s)\right)\right| d s \\
& \leq C(b-a) \sum_{l=0}^{2}\left|p^{(l)}(s)-y^{(l)}(s)\right|=\mathcal{O}\left(h^{m}\right),
\end{aligned}
$$

therefore by using the triangle inequality we have

$$
\left|\bar{z}_{\mathbf{f}}[e]\left(t_{i, j}\right)-\bar{\chi}_{\mathbf{f}}[\varepsilon]_{i, j}\right| \leq\left|\bar{z}_{\mathbf{f}}[e]\left(t_{i, j}\right)\right|+\left|\bar{\chi}_{\mathbf{f}}[\varepsilon]_{i, j}\right|=\mathcal{O}\left(h^{m}\right) .
$$

In a similar way we can find (3.5) for $k=\mathbf{v}$.

Definition 6. We define $b_{l}\left(t_{i, j}\right)$ and $c_{l}\left(t_{i, j}\right)(l=1,2, \mathbf{f}, \mathbf{v})$ as follows

$$
\begin{aligned}
& b_{1}\left(t_{i, j}\right):=\int_{0}^{1} F_{y}\left(t_{i, j}, \eta_{i, j}+\tau \varepsilon_{i, j},\left(L_{\mathcal{A}}^{(1)} \pi\right)_{i, j}, \chi^{\mathbf{f}}[\pi]_{i, j}, \chi^{\mathbf{v}}[\pi]_{i, j}\right) d \tau, \\
& b_{2}\left(t_{i, j}\right):=\int_{0}^{1} F_{y^{\prime}}\left(t_{i, j}, \eta_{i, j},\left(L_{\mathcal{A}}^{(1)} \eta\right)_{i, j}+\tau\left(L_{\mathcal{A}}^{(1)} \varepsilon\right)_{i, j}, \chi^{\mathbf{f}}[\pi]_{i, j}, \chi^{\mathbf{v}}[\pi]_{i, j}\right) d \tau, \\
& b_{\mathbf{f}}\left(t_{i, j}\right):=\int_{0}^{1} F_{z_{\mathbf{f}}}\left(t_{i, j}, \eta_{i, j},\left(L_{\mathcal{A}}^{(1)} \eta\right)_{i, j}, \chi^{\mathbf{f}}[\eta]_{i, j}+\tau \bar{\chi}^{\mathbf{f}}[\varepsilon]_{i, j}, \chi^{\mathbf{v}}[\pi]_{i, j}\right) d \tau, \\
& b_{\mathbf{v}}\left(t_{i, j}\right):=\int_{0}^{1} F_{z_{\mathbf{v}}}\left(t_{i, j}, \eta_{i, j},\left(L_{\mathcal{A}}^{(1)} \eta\right)_{i, j}, \chi^{\mathbf{f}}[\eta]_{i, j}, \chi^{\mathbf{v}}[\eta]_{i, j}+\tau \bar{\chi}^{\mathbf{v}}[\varepsilon]_{i, j}\right) d \tau, \\
& c_{1}\left(t_{i, j}\right):=\int_{0}^{1} F_{y}\left(t_{i, j}, y\left(t_{i, j}\right)+\tau e\left(t_{i, j}\right), p^{\prime}\left(t_{i, j}\right), z_{\mathbf{f}}[p]\left(t_{i, j}\right), z_{\mathbf{v}}[p]\left(t_{i, j}\right)\right) d \tau, \\
& c_{2}\left(t_{i, j}\right):=\int_{0}^{1} F_{y^{\prime}}\left(t_{i, j}, y\left(t_{i, j}\right), y^{\prime}\left(t_{i, j}\right)+\tau e^{\prime}\left(t_{i, j}\right), z_{\mathbf{f}}[p]\left(t_{i, j}\right), z_{\mathbf{v}}[p]\left(t_{i, j}\right)\right) d \tau, \\
& c_{\mathbf{f}}\left(t_{i, j}\right):=\int_{0}^{1} F_{z_{\mathbf{f}}}\left(t_{i, j}, y\left(t_{i, j}\right), y^{\prime}\left(t_{i, j}\right), z_{\mathbf{f}}[y]\left(t_{i, j}\right)+\tau \bar{z}_{\mathbf{f}}[e]\left(t_{i, j}\right), z_{\mathbf{v}}[p]\left(t_{i, j}\right)\right) d \tau, \\
& c_{\mathbf{v}}\left(t_{i, j}\right):=\int_{0}^{1} F_{z_{\mathbf{v}}}\left(t_{i, j}, y\left(t_{i, j}\right), y^{\prime}\left(t_{i, j}\right), z_{\mathbf{f}}[y]\left(t_{i, j}\right), z_{\mathbf{v}}[y]\left(t_{i, j}\right)+\tau \bar{z}_{\mathbf{v}}[e]\left(t_{i, j}\right)\right) d \tau \text {. }
\end{aligned}
$$

Lemma 11. We have

$$
\left|b_{l}\left(t_{i, j}\right)-c_{l}\left(t_{i, j}\right)\right|=\mathcal{O}(h), \quad l=1,2, \mathbf{f}, \mathbf{v} .
$$

Proof. By using the Lipschitz condition for $F_{y}, F_{y^{\prime}}, F_{z_{\mathbf{f}}}$ and $F_{z_{\mathbf{v}}}$ we can find

$$
\begin{aligned}
& \mid F_{y}\left(t_{i, j}, \eta_{i, j}+\tau \varepsilon_{i, j},\left(L_{\mathcal{A}}^{(1)} \pi\right)_{i, j}, \chi^{\mathbf{f}}[\pi]_{i, j}, \chi^{\mathbf{v}}[\pi]_{i, j}\right) \\
& \quad-F_{y}\left(t_{i, j}, y\left(t_{i, j}\right)+\tau e\left(t_{i, j}\right), p^{\prime}\left(t_{i, j}\right), z_{\mathbf{f}}[p]\left(t_{i, j}\right), z_{\mathbf{v}}[p]\left(t_{i, j}\right)\right) \mid
\end{aligned}
$$




$$
\begin{aligned}
\leq & C\left(\left|\eta_{i, j}-y\left(t_{i, j}\right)\right|+\tau\left|\varepsilon_{i, j}-e\left(t_{i, j}\right)\right|\right)+C\left|\left(L_{\mathcal{A}}^{(1)} \pi\right)_{i, j}-p^{\prime}\left(t_{i, j}\right)\right| \\
& +C\left|\chi^{\mathbf{f}}[\pi]_{i, j}-z_{\mathbf{f}}[p]\left(t_{i, j}\right)\right|+C\left|\chi^{\mathbf{v}}[\pi]_{i, j}-z_{\mathbf{v}}[p]\left(t_{i, j}\right)\right|=\mathcal{O}(h), \\
\mid F_{y^{\prime}}\left(t_{i, j}, \eta_{i, j},\left(L_{\mathcal{A}}^{(1)} \eta\right)_{i, j}+\tau\left(L_{\mathcal{A}}^{(1)} \varepsilon\right)_{i, j}, \chi^{\mathbf{f}}[\pi]_{i, j}, \chi^{\mathbf{v}}[\pi]_{i, j}\right) & \\
& -F_{y^{\prime}}\left(t_{i, j}, y\left(t_{i, j}\right), y^{\prime}\left(t_{i, j}\right)+\tau e^{\prime}\left(t_{i, j}\right), z_{\mathbf{f}}[p]\left(t_{i, j}\right), z_{\mathbf{v}}[p]\left(t_{i, j}\right)\right) \mid \\
\leq & C\left|\eta_{i, j}-y\left(t_{i, j}\right)\right|+C\left(\left|\left(L_{\mathcal{A}}^{(1)} \eta\right)_{i, j}-y^{\prime}\left(t_{i, j}\right)\right|+\tau\left|\left(L_{\mathcal{A}}^{(1)} \varepsilon\right)_{i, j}-e^{\prime}\left(t_{i, j}\right)\right|\right) \\
& +C\left|\chi^{\mathbf{f}}[\pi]_{i, j}-z_{\mathbf{f}}[p]\left(t_{i, j}\right)\right|+C\left|\chi^{\mathbf{v}}[\pi]_{i, j}-z_{\mathbf{v}}[p]\left(t_{i, j}\right)\right|=\mathcal{O}(h), \\
\mid F_{z_{\mathbf{f}}} & \left(t_{i, j}, \eta_{i, j},\left(L_{\mathcal{A}}^{(1)} \eta\right)_{i, j}, \chi^{\mathbf{f}}[\eta]_{i, j}+\tau \bar{\chi}^{\mathbf{f}}[\varepsilon]_{i, j}, \chi^{\mathbf{v}}[\pi]_{i, j}\right) \\
& -F_{z_{\mathbf{f}}}\left(t_{i, j}, y\left(t_{i, j}\right), y^{\prime}\left(t_{i, j}\right), z_{\mathbf{f}}[y]\left(t_{i, j}\right)+\tau \bar{z}_{\mathbf{f}}[e]\left(t_{i, j}\right), z_{\mathbf{v}}[p]\left(t_{i, j}\right)\right) \mid \\
\leq & C\left|\eta_{i, j}-y\left(t_{i, j}\right)\right|+C\left|\left(L_{\mathcal{A}}^{(1)} \eta\right)_{i, j}-y^{\prime}\left(t_{i, j}\right)\right|+C\left(\left|\chi^{\mathbf{f}}[\eta]_{i, j}-z_{\mathbf{f}}[y]\left(t_{i, j}\right)\right|\right. \\
& \left.+\tau\left|\bar{\chi}^{\mathbf{f}}[\varepsilon]_{i, j}-\bar{z}_{\mathbf{f}}[e]\left(t_{i, j}\right)\right|\right)+C\left|\chi^{\mathbf{v}}[\pi]_{i, j}-z_{\mathbf{v}}[p]\left(t_{i, j}\right)\right|=\mathcal{O}(h), \\
\mid F_{z_{\mathbf{v}}} & \left(t_{i, j}, \eta_{i, j},\left(L_{\mathcal{A}}^{(1)} \eta\right)_{i, j}, \chi^{\mathbf{f}}[\eta]_{i, j}, \chi^{\mathbf{v}}[\eta]_{i, j}+\tau \bar{\chi}^{\mathbf{v}}[\varepsilon]_{i, j}\right) \\
& -F_{z_{\mathbf{v}}}\left(t_{i, j}, y\left(t_{i, j}\right), y^{\prime}\left(t_{i, j}\right), z_{\mathbf{f}}[y]\left(t_{i, j}\right), z_{\mathbf{v}}[y]\left(t_{i, j}\right)+\tau \bar{z}_{\mathbf{v}}[e]\left(t_{i, j}\right)\right) \mid \\
\leq & C\left|\eta_{i, j}-y\left(t_{i, j}\right)\right|+C\left|\left(L_{\mathcal{A}}^{(1)} \eta\right)_{i, j}-y^{\prime}\left(t_{i, j}\right)\right|+C\left|\chi^{\mathbf{f}}[\eta]_{i, j}-z_{\mathbf{v}}[y]\left(t_{i, j}\right)\right| \\
& +C\left(\left|\chi^{\mathbf{v}}[\eta]_{i, j}-z_{\mathbf{v}}[y]\left(t_{i, j}\right)\right|+\tau\left|\bar{\chi}^{\mathbf{v}}[\varepsilon]_{i, j}-\bar{z}_{\mathbf{v}}[e]\left(t_{i, j}\right)\right|\right)=\mathcal{O}(h) .
\end{aligned}
$$

Now we study (3.6). For $l=1$, by using (3.7) we can get

$$
\begin{gathered}
\left|b_{1}\left(t_{i, j}\right)-c_{1}\left(t_{i, j}\right)\right| \leq \int_{0}^{1} \mid F_{y}\left(t_{i, j}, \eta_{i, j}+\tau \varepsilon_{i, j},\left(L_{\mathcal{A}}^{(1)} \pi\right)_{i, j}, \chi^{\mathbf{f}}[\pi]_{i, j}, \chi^{\mathbf{v}}[\pi]_{i, j}\right) \\
-F_{y}\left(t_{i, j}, y\left(t_{i, j}\right)+\tau e\left(t_{i, j}\right), p^{\prime}\left(t_{i, j}\right), z_{\mathbf{f}}[p]\left(t_{i, j}\right), z_{\mathbf{v}}[p]\left(t_{i, j}\right)\right) \mid d \tau=\mathcal{O}(h) .
\end{gathered}
$$

In a similar way we can find (3.6) for $l=2, \mathbf{f}$ and $\mathbf{v}$.

When $F$ is nonlinear we have the following theorem.

Theorem 5. Consider the SFVID equation (1.1) with boundary conditions (1.2), where $F\left(t, y, y^{\prime}, z_{\mathbf{f}}, z_{\mathbf{v}}\right), F_{l}\left(t, y, y^{\prime}, z_{\mathbf{f}}, z_{\mathbf{v}}\right)\left(l=y, y^{\prime}, z_{\mathbf{f}}, z_{\mathbf{v}}\right)$ are Lipschitzcontinuous. Also for nonlinear $z_{l}[\cdot](t)(l=\mathbf{f}, \mathbf{v})$ we let $K_{l}\left(t, s, u, u^{\prime}, u^{\prime \prime}\right)$ and $\left(K_{l}\right)_{j}\left(t, s, u, u^{\prime}, u^{\prime \prime}\right)\left(l=\mathbf{f}, \mathbf{v} \& j=u, u^{\prime}, u^{\prime \prime}\right)$ are Lipschitz-continuous. Assume that the SFVID problem has a unique and sufficiently smooth solution. Then the following estimate holds

$$
\|\theta\|_{\infty}=\|e-\varepsilon\|_{\infty}=\mathcal{O}\left(h^{m+1}\right),
$$

where $e$ is error, $\varepsilon$ is the error estimate and $\theta$ is the deviation of the error estimate. 
Proof. We have

$$
\begin{aligned}
& \left(L_{\mathcal{A}}^{(2)} \theta\right)_{i, j}=\left(L_{\mathcal{A}}^{(2)} e\right)_{i, j}-\left(L_{\mathcal{A}}^{(2)} \varepsilon\right)_{i, j} \\
& =\mathcal{I}_{\mathcal{A}}(\underbrace{F\left(t_{i, j}, p\left(t_{i, j}\right), p^{\prime}\left(t_{i, j}\right), z_{\mathbf{f}}[p]\left(t_{i, j}\right), z_{\mathbf{v}}[p]\left(t_{i, j}\right)\right)}_{R_{1}} \\
& -\underbrace{F\left(t_{i, j}, y\left(t_{i, j}\right), y^{\prime}\left(t_{i, j}\right), z_{\mathbf{f}}[y]\left(t_{i, j}\right), z_{\mathbf{v}}[y]\left(t_{i, j}\right)\right)}_{R_{2}}) \\
& -(\underbrace{F\left(t_{i, j}, \pi_{i, j},\left(L_{\mathcal{A}}^{(1)} \pi\right)_{i, j}, \chi^{\mathbf{f}}[\pi]_{i, j}, \chi^{\mathbf{v}}[\pi]_{i, j}\right)}_{R_{3}} \\
& -\underbrace{F\left(t_{i, j}, \eta_{i, j},\left(L_{\mathcal{A}}^{(1)} \eta\right)_{i, j}, \chi^{\mathbf{f}}[\eta]_{i, j}, \chi^{\mathbf{v}}[\eta]_{i, j}\right)}_{R_{4}}) \\
& +Q_{\mathcal{A}}\left(F\left(t_{i, j}, p\left(t_{i, j}\right), p^{\prime}\left(t_{i, j}\right), \widetilde{z}_{\mathbf{f}}[p]\left(t_{i, j}\right), \widetilde{z}_{\mathbf{v}}[p]\left(t_{i, j}\right)\right)\right) \\
& -\mathcal{I}_{\mathcal{A}}\left(F\left(t_{i, j}, p\left(t_{i, j}\right), p^{\prime}\left(t_{i, j}\right), z_{\mathbf{f}}[p]\left(t_{i, j}\right), z_{\mathbf{v}}[p]\left(t_{i, j}\right)\right)\right) \text {. }
\end{aligned}
$$

We can get

$$
\begin{aligned}
& R_{1}-R_{2}=\sum_{l=0}^{1} c_{l+1}\left(t_{i, j}\right) e^{(l)}\left(t_{i, j}\right)+\sum_{l=\mathbf{f}, \mathbf{v}} c_{l}\left(t_{i, j}\right) \bar{z}_{l}[e]\left(t_{i, j}\right), \\
& R_{3}-R_{4}=b_{1}\left(t_{i, j}\right) \varepsilon_{i, j}+b_{2}\left(t_{i, j}\right)\left(L_{\mathcal{A}}^{(1)} \varepsilon\right)_{i, j}+\sum_{l=\mathbf{f}, \mathbf{v}} b_{l}\left(t_{i, j}\right) \bar{\chi}^{l}[\varepsilon]_{i, j} .
\end{aligned}
$$

Therefore we can rewrite (3.8) as follows

$$
\begin{aligned}
\left(L_{\mathcal{A}}^{(2)} \theta\right)_{i, j} & =b_{1}\left(t_{i, j}\right) \theta_{i, j}+b_{2}\left(t_{i, j}\right)\left(L_{\mathcal{A}}^{(1)} \theta\right)_{i, j}+\sum_{l=\mathbf{f}, \mathbf{v}} b_{l}\left(t_{i, j}\right) \bar{\chi}^{l}[\theta]_{i, j} \\
& +\mathcal{I}_{\mathcal{A}}\left(c_{1}\left(t_{i, j}\right) e\left(t_{i, j}\right)+c_{2}\left(t_{i, j}\right) e^{\prime}\left(t_{i, j}\right)+\sum_{l=\mathbf{f}, \mathbf{v}} c_{l}\left(t_{i, j}\right) \bar{z}_{l}[e]\left(t_{i, j}\right)\right) \\
& -\left(c_{1}\left(t_{i, j}\right) e\left(t_{i, j}\right)+c_{2}\left(t_{i, j}\right)\left(L_{\mathcal{A}}^{(1)} e\right)_{i, j}+\sum_{l=\mathbf{f}, \mathbf{v}} c_{l}\left(t_{i, j}\right) \bar{\chi}^{l}[e]_{i, j}\right) \\
& +\left(c_{1}\left(t_{i, j}\right)-b_{1}\left(t_{i, j}\right)\right) \underbrace{e\left(t_{i, j}\right)}_{\mathcal{O}\left(h^{m}\right)}+\left(c_{2}\left(t_{i, j}\right)-b_{2}\left(t_{i, j}\right)\right) \underbrace{\left(L_{\mathcal{A}}^{(1)} e\right)_{i, j}}_{\mathcal{O}\left(h^{m}\right)} \\
& +\sum_{l=\mathbf{f}, \mathbf{v}}\left(c_{l}\left(t_{i, j}\right)-b_{l}\left(t_{i, j}\right)\right) \underbrace{\bar{\chi}^{l}[e]_{i, j}}_{\mathcal{O}\left(h^{m}\right)}+Q_{\mathcal{A}}\left(F\left(\cdot, p, p^{\prime}, \widetilde{z}_{\mathbf{f}}[p], \widetilde{z}_{\mathbf{v}}[p]\right), t_{i, j}\right) \\
& -\mathcal{I}_{\mathcal{A}}\left(F\left(\cdot, p, p^{\prime}, z_{\mathbf{f}}[p], z_{\mathbf{v}}[p]\right), t_{i, j}\right) .
\end{aligned}
$$

Then we rewrite (3.9) as follows

$$
\begin{aligned}
& \left(L_{\mathcal{A}}^{(2)} \theta\right)_{i, j}=b_{1}\left(t_{i, j}\right) \theta_{i, j}+b_{2}\left(t_{i, j}\right)\left(L_{\mathcal{A}}^{(1)} \theta\right)_{i, j}+\sum_{l=\mathbf{f}, \mathbf{v}} b_{l}\left(t_{i, j}\right) \bar{\chi}^{l}[\theta]_{i, j} \\
& +\mathcal{I}_{\mathcal{A}}\left(c_{1}\left(t_{i, j}\right) e\left(t_{i, j}\right)+c_{2}\left(t_{i, j}\right) e^{\prime}\left(t_{i, j}\right)+\sum_{l=\mathbf{f}, \mathbf{v}} c_{l}\left(t_{i, j}\right) \bar{z}_{l}[e]\left(t_{i, j}\right)\right)
\end{aligned}
$$




$$
\begin{aligned}
& -\left(c_{1}\left(t_{i, j}\right) e\left(t_{i, j}\right)+c_{2}\left(t_{i, j}\right)\left(L_{\mathcal{A}}^{(1)} e\right)_{i, j}+\sum_{l=\mathbf{f}, \mathbf{v}} c_{l}\left(t_{i, j}\right) \bar{\chi}^{l}[e]_{i, j}\right) \\
& +Q_{\mathcal{A}}\left(F\left(\cdot, p, p^{\prime}, \widetilde{z}_{\mathbf{f}}[p], \widetilde{z}_{\mathbf{v}}[p]\right), t_{i, j}\right) \\
& -\mathcal{I}_{\mathcal{A}}\left(F\left(\cdot, p, p^{\prime}, z_{\mathbf{f}}[p], z_{\mathbf{v}}[p]\right), t_{i, j}\right)+\mathcal{O}\left(h^{m+1}\right) .
\end{aligned}
$$

In a similar way to the Theorem 4 , we can complete the proof.

\section{Improvement of the deviation of the error estimate}

In particular case of (1.1)-(1.2) with

$$
z_{\mathbf{f}}[y](t)=\int_{a}^{b} K_{\mathbf{f}}(t, s, y(s)) d s, \quad z_{\mathbf{v}}[y](t)=\int_{a}^{t} K_{\mathbf{v}}(t, s, y(s)) d s
$$

we can find second order finite difference scheme. In this case by using the trapezoidal rule we can find the following finite difference scheme

$$
\begin{aligned}
& \left(L_{\mathcal{A}}^{(2)} \eta\right)_{i, j}=F\left(t_{i, j}, \eta_{i, j},\left(\bar{L}_{\mathcal{A}}^{(1)} \eta\right)_{i, j}, \varrho^{\mathbf{f}}[\eta]_{i, j}, \varrho^{\mathbf{v}}[\eta]_{i, j}\right), \quad(i, j) \in \mathcal{B}, \\
& \eta_{0,0}=r_{1}, \quad \eta_{n, 0}=r_{2},
\end{aligned}
$$

where $\left(L_{\mathcal{A}}^{(2)} \eta\right)_{i, j}$ is defined in $(2.6)$ and

$$
\begin{aligned}
\left(\bar{L}_{\mathcal{A}}^{(1)} \eta\right)_{i, j} & :=\frac{\eta_{i, j+1}-\eta_{i, j-1}}{2 \widehat{\delta}_{i, j}}, \\
\varrho^{\mathbf{f}}[\eta]_{i, j}: & =\sum_{(l, v) \in \mathcal{T}} \frac{\delta_{l, v}}{2}\left(K_{\mathbf{f}}\left(t_{i, j}, t_{l, v}, \eta_{l, v}\right)+K_{\mathbf{f}}\left(t_{i, j}, t_{l, v+1}, \eta_{l, v+1}\right)\right), \\
\varrho^{\mathbf{v}}[\eta]_{i, j} & :=\sum_{(l, v) \in \Delta_{i, j}} \frac{\delta_{l, v}}{2}\left(K_{\mathbf{v}}\left(t_{i, j}, t_{l, v}, \eta_{l, v}\right)+K_{\mathbf{v}}\left(t_{i, j}, t_{l, v+1}, \eta_{l, v+1}\right)\right) .
\end{aligned}
$$

For above case, in the same way that, as discussed in the Section 3, we can easily prove the following two theorems.

Theorem 6. Consider the SFVID equation (1.3) with (4.1) and boundary conditions (1.2). Assume that the SFVID problem has a unique and sufficiently smooth solution. Then the following estimate holds

$$
\|\theta\|_{\infty}=\|e-\varepsilon\|_{\infty}=\mathcal{O}\left(h^{m+2}\right),
$$

where $e$ is error, $\varepsilon$ is the error estimate and $\theta$ is the deviation of the error estimate.

Theorem 7. Consider the SFVID equation (1.1) with (4.1) and boundary conditions (1.2), where $F\left(t, y, y^{\prime}, z_{\mathbf{f}}, z_{\mathbf{v}}\right)$ and $F_{l}\left(t, y, y^{\prime}, z_{\mathbf{f}}, z_{\mathbf{v}}\right)\left(l=y, y^{\prime}, z_{\mathbf{f}}, z_{\mathbf{v}}\right)$ are Lipschitz-continuous. Also for nonlinear $z_{l}[\cdot](t)(l=\mathbf{f}, \mathbf{v})$ we let $K_{l}(t, s, u)$ and $\left(K_{l}\right)_{j}(t, s, u)(l=\mathbf{f}, \mathbf{v} \& j=u)$ are Lipschitz-continuous. Assume that the SFVID problem has a unique and sufficiently smooth solution. Then the following estimate holds

$$
\|\theta\|_{\infty}=\|e-\varepsilon\|_{\infty}=\mathcal{O}\left(h^{m+2}\right),
$$

where $e$ is error, $\varepsilon$ is the error estimate and $\theta$ is the deviation of the error estimate. 


\section{Numerical Examples}

In this Section we apply the numerical results. The examples 1-2 considered below are used as test for Theorem 4 and Theorem 5. Also example 3 and example 4 serve to illustrate Theorem 6 and Theorem 7 . The results obtained by using Mathematica-9 programming. Also in the examples the boundary conditions are taken from the exact solution.

Example 1. We consider the linear case as

$$
y^{\prime \prime}(t)=t y^{\prime}(t)+y(t)+a_{3}(t)+\int_{0}^{1} t s \sum_{l=0}^{2} y^{(l)}(s) d s+\int_{0}^{t} t s^{2} \sum_{l=0}^{2} y^{(l)}(s) d s .
$$

In this case $a_{3}(t)$ chosen so that exact solution is $y(t)=\exp (2 t)$. In the Table 1 we choose $m=4$ and $n$ collocation subintervals of length $1 / n$. Also in the Table 2 we choose $m=2$ and assume that $\left\{\rho_{0}=0, \rho_{1}=0.283333, \rho_{2}=\right.$ $\left.0.616667, \rho_{3}=1\right\}$.

Table 1. Numerical results for example 1.

\begin{tabular}{lllll}
\hline $\mathrm{n}$ & $\|e\|_{\infty}$ & Order & $\|\theta\|_{\infty}$ & Order \\
\hline 4 & $3.25842 \mathrm{e}-2$ & - & $3.91123 \mathrm{e}-6$ & - \\
8 & $2.11365 \mathrm{e}-6$ & 3.94637 & $1.41308 \mathrm{e}-7$ & 4.79071 \\
16 & $1.32181 \mathrm{e}-7$ & 3.99914 & $4.75659 \mathrm{e}-9$ & 4.89277 \\
32 & $8.26373 \mathrm{e}-9$ & 3.99958 & $1.54231 \mathrm{e}-10$ & 4.94676 \\
64 & $5.16669 \mathrm{e}-10$ & 3.99948 & $4.92406 \mathrm{e}-12$ & 4.96910 \\
\hline
\end{tabular}

Table 2. Numerical results for example 1.

\begin{tabular}{lllll}
\hline $\mathrm{n}$ & $\|e\|_{\infty}$ & Order & $\|\theta\|_{\infty}$ & Order \\
\hline 4 & $1.71121 \mathrm{e}-2$ & - & $2.90399 \mathrm{e}-3$ & - \\
8 & $4.54653 \mathrm{e}-3$ & 1.91218 & $4.43366 \mathrm{e}-4$ & 2.71146 \\
16 & $1.15124 \mathrm{e}-3$ & 1.98158 & $6.15327 \mathrm{e}-5$ & 2.84907 \\
32 & $2.89467 \mathrm{e}-4$ & 1.99172 & $8.23059 \mathrm{e}-6$ & 2.90229 \\
64 & $7.25721 \mathrm{e}-5$ & 1.99591 & $1.09647 \mathrm{e}-6$ & 2.90814 \\
\hline
\end{tabular}

Example 2. We consider the problem

$$
\begin{aligned}
y^{\prime \prime}(t) & =t y^{\prime}(t)+y^{2}(t)+a_{3}(t)+\int_{0}^{1} t s\left(y(s)+y^{\prime}(s)+\left(y^{\prime \prime}(s)\right)^{2}\right) d s \\
& +\int_{0}^{t} t s^{2}\left(y(s) y^{\prime}(s)+y^{\prime \prime}(s)\right) d s
\end{aligned}
$$

$a_{3}(t)$ chosen so that exact solution is $y(t)=\sin 2 t$. For this example we choose $n$ collocation subintervals of length $1 / n$. In the Table 3 we choose $m=4$ and assume that $\rho_{i}(i=0, \ldots, 5)$ are equidistant points. In the Table 4 we choose $m=3$ and $\left\{\rho_{0}=0, \rho_{1}=0.21, \rho_{2}=0.46, \rho_{3}=0.71, \rho_{4}=1\right\}$. 
Table 3. Numerical results for example 2 .

\begin{tabular}{lllll}
\hline $\mathrm{n}$ & $\|e\|_{\infty}$ & Order & $\|\theta\|_{\infty}$ & Order \\
\hline 2 & $5.65455 \mathrm{e}-7$ & - & $9.43288 \mathrm{e}-8$ & - \\
4 & $3.21333 \mathrm{e}-8$ & 4.13727 & $3.58181 \mathrm{e}-9$ & 4.71894 \\
8 & $2.02451 \mathrm{e}-9$ & 3.98843 & $1.21604 \mathrm{e}-10$ & 4.88043 \\
16 & $1.25615 \mathrm{e}-10$ & 4.01049 & $3.94984 \mathrm{e}-12$ & 4.94425 \\
\hline
\end{tabular}

Table 4. Numerical results for example 2.

\begin{tabular}{lllll}
\hline $\mathrm{n}$ & $\|e\|_{\infty}$ & Order & $\|\theta\|_{\infty}$ & Order \\
\hline 2 & $1.20478 \mathrm{e}-5$ & - & $4.38700 \mathrm{e}-6$ & - \\
4 & $1.58034 \mathrm{e}-6$ & 2.93046 & $2.48796 \mathrm{e}-7$ & 4.14020 \\
8 & $2.02665 \mathrm{e}-7$ & 2.96307 & $1.45576 \mathrm{e}-8$ & 4.09512 \\
16 & $2.57390 \mathrm{e}-8$ & 2.97707 & $8.12608 \mathrm{e}-10$ & 4.16307 \\
\hline
\end{tabular}

Example 3. In this example we consider here the following linear problem

$$
y^{\prime \prime}(t)=\cos (t) y^{\prime}(t)+y(t)+a_{3}(t)+\int_{0}^{1} y(s) d s+\int_{0}^{t} t \cos (s) y(s) d s,
$$

$a_{3}(t)$ chosen so that exact solution is $y(t)=t \sin (t)$. For this example we choose $n$ collocation subintervals of length $1 / n$. In the Table 5 we choose $m=2$ and assume that $\rho_{i}(i=0, \ldots, 3)$ are equidistant points. Also in the Table 6 , we consider $m=3$ and $\left\{\rho_{0}=0, \rho_{1}=0.2, \rho_{2}=0.55, \rho_{3}=0.72, \rho_{4}=1\right\}$.

Table 5. Numerical results for example 3 .

\begin{tabular}{lllll}
\hline $\mathrm{n}$ & $\|e\|_{\infty}$ & Order & $\|\theta\|_{\infty}$ & Order \\
\hline 2 & $2.26647 \mathrm{e}-3$ & - & $8.45242 \mathrm{e}-6$ & - \\
4 & $5.63358 \mathrm{e}-4$ & 2.00832 & $4.92246 \mathrm{e}-7$ & 4.10191 \\
8 & $1.40539 \mathrm{e}-4$ & 2.00308 & $2.73140 \mathrm{e}-8$ & 4.17167 \\
16 & $3.51146 \mathrm{e}-5$ & 2.00083 & $1.61489 \mathrm{e}-9$ & 4.08014 \\
\hline
\end{tabular}

Table 6. Numerical results for example 3 .

\begin{tabular}{lllll}
\hline $\mathrm{n}$ & $\|e\|_{\infty}$ & Order & $\|\theta\|_{\infty}$ & Order \\
\hline 2 & $1.25407 \mathrm{e}-5$ & - & $4.98589 \mathrm{e}-7$ & - \\
4 & $2.63666 \mathrm{e}-6$ & 2.24984 & $1.47117 \mathrm{e}-8$ & 5.08282 \\
8 & $4.24484 \mathrm{e}-7$ & 2.63493 & $4.31918 \mathrm{e}-10$ & 5.09006 \\
16 & $6.00350 \mathrm{e}-8$ & 2.82183 & $7.28798 \mathrm{e}-12$ & 5.88910 \\
\hline
\end{tabular}

Example 4. As a last study we consider the nonlinear case as

$$
y^{\prime \prime}(t)=y^{\prime}(t)+y^{2}(t)+a_{3}(t)+\left(\int_{0}^{1} \sin (t) y(s) d s\right)\left(\int_{0}^{t} \cos (s) y(s) d s\right),
$$


$a_{3}(t)$ chosen so that exact solution is $y(t)=\sin (t)$. In this example for Table 7 we choose $m=2$ and assume that $\tau_{i}$ are equidistant point. Also In this table we choose equidistant points for $\rho_{i}$. In the Table 8 we choose $m=3$ and $\left\{\rho_{0}=0, \rho_{1}=0.23, \rho_{2}=0.55, \rho_{3}=0.78, \rho_{4}=1\right\}$.

Table 7. Numerical results for example 4.

\begin{tabular}{lllll}
\hline $\mathrm{n}$ & $\|e\|_{\infty}$ & Order & $\|\theta\|_{\infty}$ & Order \\
\hline 2 & $3.68252 \mathrm{e}-4$ & - & $1.38395 \mathrm{e}-6$ & - \\
4 & $8.78560 \mathrm{e}-5$ & 2.06748 & $5.77515 \mathrm{e}-8$ & 4.58279 \\
8 & $2.28186 \mathrm{e}-5$ & 1.94493 & $2.29570 \mathrm{e}-9$ & 4.65285 \\
16 & $5.68809 \mathrm{e}-6$ & 2.00419 & $1.00123 \mathrm{e}-10$ & 4.51909 \\
\hline
\end{tabular}

Table 8. Numerical results for example 4.

\begin{tabular}{lllll}
\hline $\mathrm{n}$ & $\|e\|_{\infty}$ & Order & $\|\theta\|_{\infty}$ & Order \\
\hline 2 & $1.80270 \mathrm{e}-6$ & - & $9.53181 \mathrm{e}-8$ & - \\
4 & $2.48418 \mathrm{e}-7$ & 2.85931 & $1.52154 \mathrm{e}-9$ & 5.96915 \\
8 & $3.46815 \mathrm{e}-8$ & 2.84053 & $2.39238 \mathrm{e}-11$ & 5.99094 \\
16 & $4.55469 \mathrm{e}-9$ & 2.92874 & $7.47957 \mathrm{e}-13$ & 4.99934 \\
\hline
\end{tabular}

\section{Conclusions}

In this paper, we have constructed efficient asymptotically correct a posteriori error estimates for the numerical approximation of second order Fredholm Volterra integro differential equations. Also it is shown that when we use $m$ degree piecewise polynomial collocation method, the order of the deviation of the error estimation is $\mathcal{O}\left(h^{m+1}\right)$. In the previous section, numerical examples confirming the theoretical results are given.

\section{References}

[1] W. Auzinger, O. Koch and A. Saboor Bagherzadeh. Error estimation based on locally weighted defect for boundary value problems in second order ordinary differential equations. BIT. Numer. Math., 54(4):873-900, 2014. http://dx.doi.org/10.1007/s10543-014-0488-y.

[2] W. Auzinger, O. Koch, D. Praetorius and E. Weinmller. New a posteriori error estimates for singular boundary value problem. Numer. Algorithms., 40(1):79100, 2005. http://dx.doi.org/10.1007/s11075-005-3791-5.

[3] A. Saboor Bagherzadeh. Defect-based error estimation for higher order differential equations. PhD thesis, Vienna University of Technology, 2011.

[4] K. Bohmer, P. Hemker and H.J. Stetter. The defect correction approach. Computing, Suppl., 5:1-32, 1984. http://dx.doi.org/10.1007/978-3-7091-7023-6_1. 
[5] H. Brunner. Collocation Methods for Volterra Integral and Related Functional Differential Equations. Cambridge University Press, 2004. http://dx.doi.org/10.1017/CBO9780511543234.

[6] R.J. Hangelbroek, H.G. Kaper and G.K. Leaf. Collocation methods for Volterra integral and related functional differential equations. Siam J. Numer. Anal., 14(1):377-390, 1977. http://dx.doi.org/10.1137/0714023.

[7] I. Parts, A. Pedas and E. Tamme. Piecewise polynomial collocation for Fredholm integro-differential equations with weakly singular kernels. SIAM J. Numer. Anal., 41(5):1897-1911, 2005. http://dx.doi.org/10.1137/040612452.

[8] A. Pedas and E. Tamme. Spline collocation method for integro-differential equations with weakly singular kernels. J. Comput. Appl. Math., 197(1):253-269, 2006. http://dx.doi.org/10.1016/j.cam.2005.07.035.

[9] H.J. Stetter. The defect correction principle and discretization methods. Numer. Math., 29(4):425-443, 1978. http://dx.doi.org/10.1007/BF01432879.

[10] J. Stoer and R. Bulirsch. Introduction to numerical analysis, volume 12. Third edition, Springer-Verlg, 2002. http://dx.doi.org/10.1007/978-0-387-21738-3.

[11] M. Turkyilmazoglu. An effective approach for numerical solutions of high-order Fredholm integro-differential equations. Appl. Math. Comput., 227:384-398, 2014. http://dx.doi.org/10.1016/j.amc.2013.10.079.

[12] M. Turkyilmazoglu. High-order nonlinear Volterra-Fredholm-Hammerstein integro-differential equations and their effective computation. Appl. Math. Comput., 247:410-416, 2014. http://dx.doi.org/10.1016/j.amc.2014.08.074. 